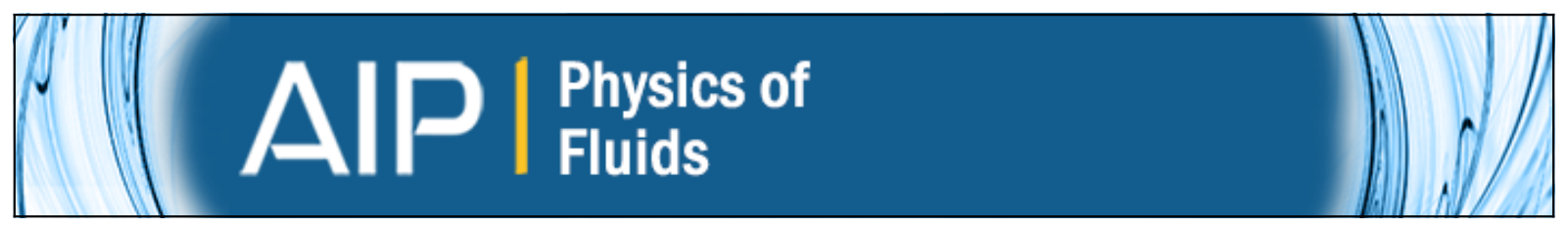

The emergence of jets and vortices in freely evolving, shallowwater turbulence on a sphere

James YK. Cho and Lorenzo M. Polvani

Citation: Physics of Fluids (1994-present) 8, 1531 (1996); doi: 10.1063/1.868929

View online: http://dx.doi.org/10.1063/1.868929

View Table of Contents: http://scitation.aip.org/content/aip/journal/pof2/8/6?ver=pdfcov

Published by the AIP Publishing

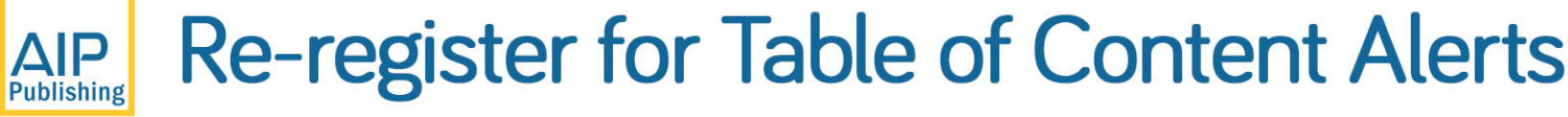

\section{Create a profile.}




\title{
The emergence of jets and vortices in freely evolving, shallow-water turbulence on a sphere
}

\author{
James Y-K. Cho a) and Lorenzo M. Polvani ${ }^{\mathrm{b})}$ \\ Program in Applied Mathematics and Department of Applied Physics, Columbia University, \\ New York, New York 10027
}

(Received 1 June 1995; accepted 26 December 1995)

\begin{abstract}
Results from a series of simulations of unforced turbulence evolving within a shallow layer of fluid on a rotating sphere are presented. Simulations show that the turbulent evolution in the spherical domain is strongly dependent on numerical and physical conditions. The independent effects of (1) (hyper)dissipation and initial spectrum, (2) rotation rate, and (3) Rossby deformation radius are carefully isolated and studied in detail. In the nondivergent and nonrotating case, an initially turbulent flow evolves into a vorticity quadrupole at long times, a direct consequence of angular momentum conservation. In the presence of sufficiently strong rotation, the nondivergent long-time behavior yields a field dominated by polar vortices-as previously reported by Yoden and Yamada. In contrast, the case with a finite deformation radius (i.e., the full spherical shallow-water system) spontaneously evolves toward a banded configuration, the number of bands increasing with the rotation rate. A direct application of this shallow-water model to the Jovian atmosphere is discussed. Using standard values for the planetary radius and rotation, we show how the initially turbulent flow self-organizes into a potential vorticity field containing zonal structures, where regions of steep potential vorticity gradients (jets) separate relatively homogenized bands. Moreover, Jovian parameter values in our simulations lead to a strong vorticity asymmetry, favoring anticyclonic vortices-in further agreement with observations. (C) 1996 American Institute of Physics. [S1070-6631(96)02004-9]
\end{abstract}

\section{INTRODUCTION}

In recent years, much progress has been made in the study of high Reynolds number, two-dimensional (2-D) turbulence via direct numerical integration. The computational approach provides a direct test of theoretical predictions and offers a first step toward understanding turbulent phenomena relevant to atmospheric and ocean dynamics. However, except for a few investigations, past 2-D turbulence simulations have been mainly restricted to doubly periodic planar domains with rigid surface, often without differential rotation. While useful for studying scaling-symmetry breaking due to coherent structure formation or inverse cascade arrest due to anisotropic conditions, the rigid surface and planar restrictions are severely limiting for geophysical applications. In this paper, we proceed one step further and present results from simulations of rotating shallow-water decaying turbulence in spherical geometry.

The shallow-water model represents a thin, homogeneous layer of fluid with a free surface, which moves under the influence of gravitational and Coriolis forces. The model in spherical geometry incorporates the effects of both differential rotation ( $\beta$ effect) and stratification (through a finite deformation radius) and is the simplest of the commonly used atmospheric dynamics models. Due to its higher complexity, the shallow-water system in spherical geometry allows a much greater variety of physical phenomena than the planar 2-D model. In this paper, we focus on the roles of simulation conditions, differential rotation due to spherical

\footnotetext{
${ }^{a)}$ Electronic mail: jcho@appmath.columbia.edu

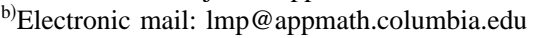

geometry, and free surface variations. Specifically, we are interested in their respective roles on the morphology of selforganizing physical structures that spontaneously arise in the turbulent shallow-water system.

Owing to differences in emphasis between the geophysical and theoretical turbulence communities, a somewhat confusing nomenclature is found in the literature. In order to clarify the terminology and locate the present work with respect to previous studies, a number of relevant cases and their associated physical parameters are summarized in Table I (the role played by the different parameters, as will become clear, is discussed in the ensuing sections and is the primary concern of this paper). For the case of planar 2-D turbulence, a wealth of investigations is available, ${ }^{1-7}$ while its spherical counterpart has received little attention. ${ }^{8-10}$ Similarly, $\beta$-plane turbulence has been well studied ${ }^{11-14}$ (though, almost exclusively under forced conditions), while the rotating spherical case has only been recently numerically simulated. ${ }^{8,15,16,9}$ As for shallow water, only three studies of the $f$ plane case are available. ${ }^{17-19}$ To the best of our knowledge, there has been no past study of shallow-water turbulence in the spherical geometry. This work is partially aimed at filling this gap.

Moreover, even in the 2-D cases confined to the plane, most of the turbulence simulations to date have not explored thoroughly the dependence of the evolution on the numerical dissipation and initial conditions, making comparisons of results somewhat difficult. ${ }^{20}$ In freely evolving turbulence, a careful exploration is critical, since this dependence seems to be more acute than in forced situations. For instance, Santangelo et al. ${ }^{5}$ have found striking differences in the evolution due to the steepness of the initial spectrum. Their initial 
TABLE I. Here $f \equiv 2 \Omega \sin \vartheta$ is the Coriolis parameter; $\Omega$ is the rotation rate and $\vartheta$ is the latitude; $\beta$ is the latitudinal gradient of $f ; \delta \equiv \nabla \cdot \mathbf{v}$ is the velocity divergence; $L_{D} \equiv \sqrt{g H} / 2 \Omega$ is the Rossby deformation radius, and $R \equiv U / 2 \Omega L$ is the Rossby number-where $H, U, L$ are the characteristic height, velocity, and length, respectively.

\begin{tabular}{|c|c|c|c|c|}
\hline & \multicolumn{4}{|c|}{ Physical complexity } \\
\hline \multirow{4}{*}{ GEOMETRY } & & $\begin{array}{l}\text { Nondivergent, } \\
\text { nonrotating } \\
(\delta=0, \Omega=0)\end{array}$ & $\begin{array}{l}\text { Nondivergent, } \\
\text { rotating } \\
(\delta=0, \Omega \neq 0)\end{array}$ & $\begin{array}{l}\text { Divergent, } \\
\text { rotating } \\
(\delta \neq 0, \Omega \neq 0)\end{array}$ \\
\hline & & $f=0$ & $\beta=$ const & $f=\mathrm{const} ; \beta=0$ \\
\hline & Plane & $\begin{array}{l}H, L_{D}, R \rightarrow \infty \\
\text { "planar 2-D" }\end{array}$ & $\begin{array}{c}H, L_{D} \rightarrow \infty ; R<\infty \\
\text { " } \beta \text {-plane } 2 \text {-D" }\end{array}$ & $\begin{array}{l}H, L_{D}, R<\infty \\
\text { "f-plane SW", }\end{array}$ \\
\hline & Sphere & $\begin{array}{c}f=0 \\
H, L_{D}, R \rightarrow \infty \\
\text { "spherical 2-D", }\end{array}$ & $\begin{array}{l}\beta=\frac{2 \Omega}{a} \cos \vartheta \\
H, L_{D} \rightarrow \infty ; R<\infty \\
\text { "rot. sph. 2-D"' }\end{array}$ & $\begin{array}{l}\beta=\frac{2 \Omega}{a} \cos \vartheta \\
H, L_{D}, R<\infty \\
\text { "rot. sph. SW" }\end{array}$ \\
\hline
\end{tabular}

finding clearly demonstrates the need for performing a thorough simulation-parameter sensitivity study to ascertain which behaviors are a direct consequence of parameter choices.

In view of this, our paper is organized in the following way. After a brief description of the physical models and the numerical procedure in Sec. II, we start in Sec. III by studying the simplest possible spherical system (the nondivergent, nonrotating case of Table I), in order to clearly characterize the dependence of the evolution on the hyperdissipation, initial spectrum, and spherical geometry. Readers interested in more physical effects may skip this section. Having carefully accessed the sensitivity to simulation conditions, we increment the physical complexity of the system by introducing rotation and describe its effects on the flow evolution in Sec. IV. In Sec. V, we allow for the variations in the height of the fluid layer (i.e., vortex-tube stretching) and consider the additional effects of removing the nondivergence constraint under differential rotation. Having studied each physical effect in detail, we demonstrate one direct application using Jovian parameters in Sec. VI. Our conclusions are then presented in Sec. VII.

\section{PHYSICAL MODELS AND NUMERICAL PROCEDURE}

We begin by briefly reviewing the shallow-water system and its relationship to the simpler, nondivergent models (see Table I), which are also considered in this paper. It is well known that a thin layer of homogeneous (constant density), hydrostatically balanced, rotating fluid with a free surface is governed by the system of shallow-water equations (SWE). ${ }^{21}$ In general coordinates, the SWE have the following form:

$$
\begin{aligned}
& \partial_{t} \mathbf{v}+\mathbf{v} \cdot \boldsymbol{\nabla} \mathbf{v}=-g \nabla h-f \mathbf{k} \times \mathbf{v}, \\
& \partial_{t} h+\mathbf{v} \cdot \boldsymbol{\nabla} h=-h \boldsymbol{\nabla} \cdot \mathbf{v},
\end{aligned}
$$

where $\mathbf{v}(\mathbf{x}, t)$ is the horizontal velocity and $h=h(\mathbf{x}, t)$ is the free surface height. The variable $f$ is the Coriolis parameter, proportional to the rotation rate, and $g$ is the gravitational acceleration.
Physically, given the characteristic scales of length, velocity, and height ( $L, U$, and $H$, respectively), one can define the Rossby number and the Froude number,

$$
R \equiv \frac{U}{2 \Omega L} \quad \text { and } F \equiv \frac{U}{\sqrt{g H}},
$$

respectively, with $\Omega$ representing the rotation rate. These quantities define the physically relevant $(R, F)$ parameter space for the SWE. Another useful nondimensional quantity is the Burger number:

$$
B \equiv \frac{R^{2}}{F^{2}}=\left(\frac{L_{D}}{L}\right)^{2},
$$

where $L_{D} \equiv \sqrt{g H} / 2 \Omega$ is the Rossby deformation radius. With these definitions, one can obtain a general set of nondimensional equations,

$$
\begin{aligned}
& \partial_{t} \mathbf{v}^{\prime}+\mathbf{v}^{\prime} \cdot \boldsymbol{\nabla} \mathbf{v}^{\prime}=-B R^{-1} \nabla h^{\prime}-R^{-1} \mathbf{k} \times \mathbf{v}^{\prime}, \\
& \partial_{t} h^{\prime}+\mathbf{v}^{\prime} \cdot \boldsymbol{\nabla} h^{\prime}=-\left(1+h^{\prime}\right) \boldsymbol{\nabla} \cdot \mathbf{v}^{\prime},
\end{aligned}
$$

where the following scaling has been used:

$$
(\mathbf{v}, h)=\left[U \mathbf{v}^{\prime}, H\left(1+h^{\prime}\right)\right],
$$

such that $h^{\prime} \equiv \widetilde{h} / H$ is the nondimensional characteristic deviation from the mean height; $\widetilde{h}$ is the dimensional deviation. Note that, as we have defined it, $h^{\prime}$ need not be small.

With the above set of nondimensional equations, the relationship between the shallow-water system and the simpler cases may easily be seen. When $h^{\prime}=0$ (i.e., either $\widetilde{h} \rightarrow 0$ or $H \rightarrow \infty$ ) Eqs. (5) - (7) immediately reduce to the rotating 2-D case for which $L_{D} \rightarrow \infty$ (cf. the nondivergent, rotating column of Table I) and the fluid surface is rigid. In addition, in the limit $R \rightarrow \infty$, the nonrotating case is obtained (cf. the nondivergent, nonrotating column of Table I). The nondivergent equations for nonrotating and rotating situations in their more common dimensional vorticity-streamfunction form are, respectively,

$$
\partial_{t} \zeta+J\{\psi, \zeta\}=0
$$

and

$$
\partial_{t} \zeta+J\{\psi, \zeta+f\}=0,
$$


where $J\{\cdot, \cdot\}$ is the Jacobian operator, $\psi$ is the streamfunction, $\zeta=\Delta \psi=\mathbf{k} \cdot \boldsymbol{\nabla} \times \mathbf{v}$ is the relative vorticity, and $\zeta+f \equiv \zeta_{a}$ is the absolute vorticity.

One of the objectives of this paper is to study the combined effects of a finite deformation radius and the $\beta$ effect. This cannot be accomplished in the doubly periodic planar domain since gravity waves would experience jump discontinuities in the phase speed at the edges of the domain. Hence, unless one is willing to accept the limitations of asymptotically approximating the SWE, ${ }^{22}$ the planar geometry must be abandoned, and the obvious one to choose is the sphere. On the sphere, the components of $\mathbf{v}, u=u(\lambda, \vartheta, t)$ and $v=v(\lambda, \vartheta, t)$, represent the eastward and the northward velocities, respectively, at the location $(\lambda, \vartheta) \Rightarrow$ (longitude, latitude), while $h=h(\lambda, \vartheta, t)$ is the surface height of the thin shell of fluid wrapping the sphere of radius $a$, such that $h \ll a$. The Coriolis parameter, $f=2 \Omega \sin \vartheta$.

In the absence of dissipation, the SWE possess a number of useful invariants: (1) the total height, $H=\langle h\rangle^{23}$ (conservation of mass); (2) the total energy $\mathscr{E}=\left\langle\frac{1}{2}\left(u^{2}+v^{2}\right) h\right.$ $\left.+\frac{1}{2} g h^{2}\right\rangle$, where $\mathscr{E}$ is the sum of the potential and kinetic energies, respectively; and (3) the potential enstrophy $\left\langle\frac{1}{2} \zeta_{a}^{2} h\right\rangle$. The potential vorticity, $q \equiv \zeta_{a} / h$, is a material invariant in the shallow-water system. In the nondivergent ( $h$ $=$ const $)$ and the nonrotating $(f=0)$ cases, these expressions for the invariants reduce accordingly. We note that, due to the nonquadratic nature of the energy and enstrophy invariants, analytical theories (e.g., closure schemes) are much more difficult to construct for the SWE than in the nondivergent situations.

To numerically integrate the equation(s) governing the dynamics in each of the spherical situations in Table I, a pseudospectral algorithm that projects the field variables into a space of spherical harmonics via the Gauss-Legendre transform is used. ${ }^{24}$ The time stepping is performed using a second-order accurate leapfrog scheme with a RobertAsselin filter, which can be used to control modal splitting. ${ }^{25}$ A triangular shape truncation in spectral, $(m, n)$, space with up to 170 resolved $n$ modes (denoted T170) is employed; here, $m$ and $n$ are the zonal and the total wave numbers, respectively. ${ }^{26}$ The truncation results in an initial spectral energy density $E(n, t=0)$ that is equipartitioned among the $m$ modes in each of the $n$ modes spanned by a triangular mask in spectral space. The kinetic energy density is related to the vorticity field, $\zeta=\zeta(\lambda, \vartheta, t)$, by

$$
E(n, t)=\frac{1}{2} \sum_{m=-n}^{n} \frac{a^{2}}{n(n+1)}\left|\zeta_{n}^{m}(t)\right|^{2},
$$

where $\zeta_{n}^{m}(t)$ are the coefficients in the truncated, spherical harmonic expansion of $\zeta$ :

$$
\zeta(\lambda, \vartheta, t)=\sum_{n=1}^{n_{t}} \sum_{m=-n}^{n} \zeta_{n}^{m}(t) P_{n}^{m}(\vartheta) e^{i m \lambda},
$$

where $P_{n}^{m}$ are the standard associated Legendre polynomials.

Other dynamical variables can be self-consistently related via the Helmholtz theorem, in which $\mathbf{v}=\hat{\mathbf{r}} \times \boldsymbol{\nabla} \psi+\nabla \chi$ is linearly decomposed into a rotational part involving the streamfunction, $\psi$, and a divergent part involving the veloc- ity potential, $\chi$, such that the vorticity $\zeta \equiv \hat{\mathbf{r}} \cdot \nabla \times \mathbf{v}=\Delta \psi$ and the divergence $\delta \equiv \nabla \cdot \mathbf{v}=\Delta \chi$. Given the truncation number, a grid large enough (e.g., T170 $\Rightarrow 512 \times 256$ grid) is used in order to evaluate the transform integrals exactly and nullify aliasing errors to within machine accuracy. ${ }^{27}$

Finally, a word about our choice of scales. The characteristic length scale, $L$, is chosen to be $\pi a / 2 n_{0}$, where $n_{0}$ is the most-energetic scale. Throughout the paper, time is scaled with an advective time scale, $T \equiv a / U$, where $U$ $\equiv \sqrt{2 \mathscr{E}}$; this is uniformly chosen for all the runs rather than the enstrophy-based, vortex turnaround time commonly adopted in planar computations. The latter time scale can vary significantly from run to run under different physical conditions and within the duration of a single run.

\section{SENSITIVITY TO SIMULATION CONDITIONS}

Almost without exception, past turbulence simulations have been performed using a single choice of dissipation and/or initial condition without providing elements to assess the degree to which their results (e.g., values of spectral slopes, self-similarity claims, etc.) may depend on their choices. In this paper, in order to clearly delineate this "numerical sensitivity" issue from the effects of the more complicated physical systems of Secs. IV and V, we begin by confining the system to the simplest possible situation with numerical dissipation-i.e., the nondivergent, nonrotating system (see Table I) governed by the equation

$$
\partial_{t} \zeta+J\{\psi, \zeta\}=(-1)^{p+1} \nu_{2 p} \Delta^{p} \zeta,
$$

where $J\{\cdot, \cdot\}$ is now the spherical Jacobian operator.

To isolate the sensitivity of the evolution due to the form of the dissipation, the power of the dissipation operator, $p$, in Eq. (12), is varied while adjusting the diffusion coefficient, $\nu_{2 p}$, accordingly. Given all the other parameters in a run, the smallest possible value of $\nu_{2 p}$ is obtained empirically via a preliminary set of runs, in which $\nu_{2 p}$ is initially chosen so that $\nu_{2 p}\left[n_{t}\left(n_{t}+1\right) / a^{2}\right]^{p}=1$, where $n_{t}$ is the truncation (maximally resolved) scale. Note the geometrical factor of $a^{2}$; from here on the value of $\nu_{2 p}$ will be given in units of $a^{2 p}$ for easier comparison with planar cases.

As for the initial condition, the same functional form is used for all the runs presented in this paper. At $t=0$ the following energy spectral density distribution is specified:

$$
E(n, 0)=\frac{\mathscr{C n} n^{\gamma / 2}}{\left(n+n_{0}\right)^{\gamma}} .
$$

The value of $\mathscr{b}$ is directly related to the velocity scale $U$, while the parameters, $n_{0}$ and $\gamma$, are used to control, respectively, the peak location and the width of the spectrum. Each mode is initialized with a random phase. The spectra for several different values of $\gamma$ and $n_{0}$ are shown in Fig. 1.

Figure 2(a) illustrates a typical physical space evolution of an initially random vorticity field at high resolution (T170) with $\left(p, \nu_{2 p}, n_{0}, \gamma\right)=\left(8,3.0 \times 10^{-35}, 7,20\right)$. In Fig. 2(b), the spectral space evolution of the same run at three representative times, $t=(0,1,9)$, corresponding to initial, early, and late times is shown. Here the time $t=1$ corresponds to approximately ten vortex turnaround times. 

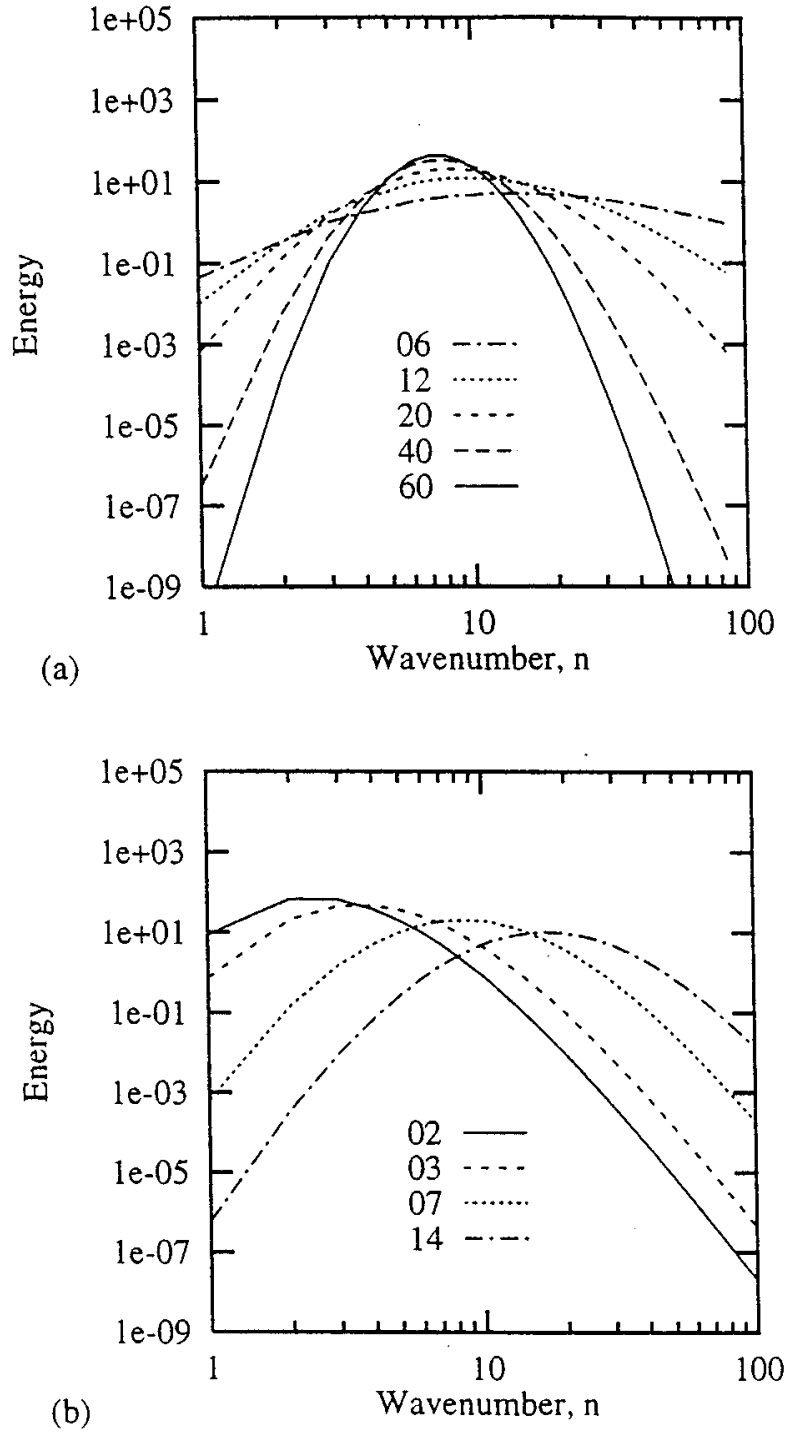

FIG. 1. Initial spectra for several parameter values of (a) the steepness, $\gamma=\{6,12,20,40,60\}$ and (b) the peak wave number, $n_{0}=\{2,3,7,14\}$. The total energy in each case is identical.

It is clear from these figures that the long-time evolution is qualitatively similar to those in high-resolution, decaying planar computations. In particular, note the following features: (1) the early creation of high-gradient, filamentary structures $[t=[1: 2]$ in Fig. 2(a)] and the beginning of the inverse cascade $[t=1$ in Fig. 2(b)]; and (2) the eventual emergence of long-lived coherent structures $[t=[4: 6]$ in Fig. 2(a)], with the corresponding steepening of the inertial range $[t=9$ in Fig. 2(b)]. These features are general and are independent of planar or spherical geometry, as was previously shown in the lower resolution, shorter integration time, spectral computations of Yoden and Yamada. ${ }^{9}$

On the sphere, however, a very long-time integration shows that coherent structures ultimately evolve to a vortical-quadrupole state-rather than a dipole state, as on a plane. ${ }^{28}$ Figure 3 depicts a typical very long-time run, in which the quadrupole configuration is reached. Once reached, the basic configuration is unchanged, even after 70 advective times; no further reduction in the number of vortices occur, even during several close encounters by the constituent vortices over this long, dynamically active period. We point out that here only $0.3 \%$ of the total energy is lost at the end of the run with no loss occurring after $t=10$. The quadrupole configuration is a direct consequence of angular momentum conservation on the sphere, which forbids the flux of energy in or out of the $n=1$ mode, thus forcing a pile-up at the $n=2$ mode; the early manifestation of this is clearly visible in Fig. 2(b) (contrast this with Fig. 2 in Ref. 1, corresponding to a planar calculation).

Having illustrated the general features of a typical highresolution spectral calculation, we now consider the effects of varying the power, $p$, of the dissipation operator. Figure 4 shows the spectra at $t=1$ for runs using $p=\{1,2,4,8\}$. All the runs begin with a common initial condition, which is identical to that of the run depicted in Fig. 2. Even at this representative "early" time (chosen to minimize the coherent structure-associated effects on the spectra), two nontrivial trends in the spectral behavior can be detected: (1) the use of hyperdissipation has the effect of extending the inertial range, and (2) the inertial range slope $x$ [such that $E(n) \sim n^{x}$ for $\left.1 \ll n<n_{t}\right]$ appears less steep with increasing $p$ with the inertial ranges "converging" toward the one corresponding to $p=8$. Here, we measure the slopes to range from -4.0 \pm 0.1 at $p=1$ to $-3.3 \pm 0.1$ at $p=8$ [see the $x(1)$ column of Table II] with the uncertainty reflecting our inability to accurately ascertain the true extent of the inertial range. ${ }^{29}$

Both trends can be easily explained by the activity present in the corresponding physical space, shown in Fig. 5. A higher-powered dissipation operator allows more spacefilling, filamentary structures to be formed and sustained. As has been shown in the two recent studies of simpler, vortex dynamics problems, ${ }^{30,31}$ the larger $p$ values lead to less dissipation and sharper small-scale vorticity gradients. We quantify this effect by the palinstrophy, $\mathscr{P}(t) \equiv\left\langle\frac{1}{2}(\nabla \zeta)^{2}\right\rangle$ $=\sum_{n=1}^{n_{t}} n^{4} E(n, t)$; see Table II. As shown in Fig. 6, these early differences lead to a substantial deviation in the subsequent vortex population at later times; the energy spectra (not shown) for the two runs are also correspondingly different.

Next, we proceed to describing the effects of the shape of prescribed initial spectrum on the turbulent evolution. Figure 7 (a) summarizes the results for different initial spectral steepness, $\gamma=\{6,12,20,40,60\}$, as illustrated in Fig. 1(a). The spectra in Fig. 7(a) are shown at time, $t=9$, corresponding to the "late" stage of evolution when the coherent structures have existed for many time periods and the enstrophy decay behavior is flat in all the runs. The parameters for these runs are $\left(p, \nu_{2 p}, n_{0}\right)=\left(4,3.0 \times 10^{-17}, 7\right)$. As can be seen, the inertial range slope does not seem to be strongly affected by the different initial steepness, even though the initial total enstrophies differ by a factor of nearly 20, due to the different values of $\gamma$. This behavior clarifies that reported in Ref. 5, in which a similar difference of initial enstrophy (a factor of 15 in that study) led to a dramatic difference in the inertial range. Our study suggests that the strong dependence on the inertial range slope in their case is not due to initial steepness 

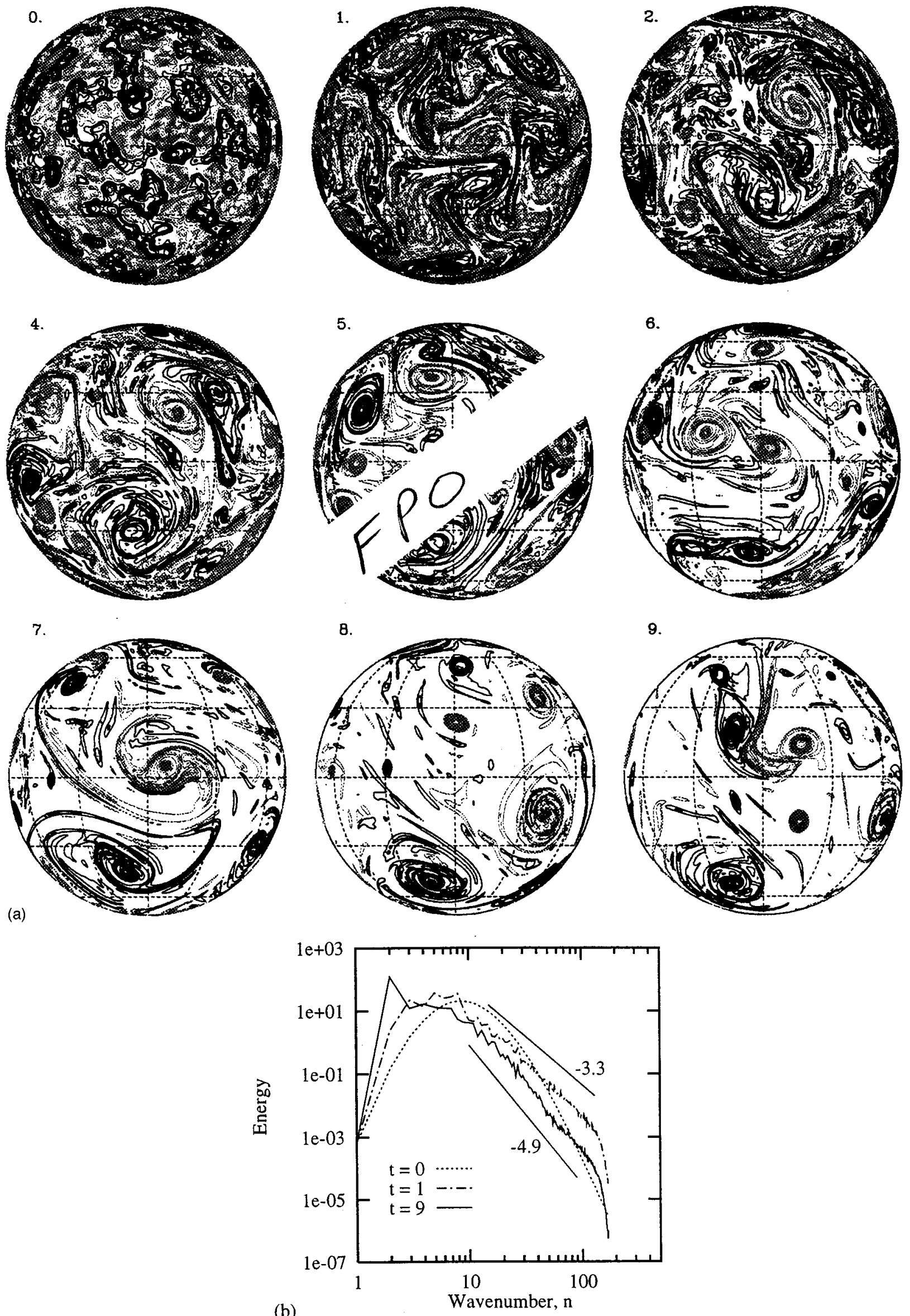

FIG. 2. Typical 2-D evolution at a T170 resolution: (a) vorticity contours at times marked at the upper left of each frame, positive values are in red and negative values are in blue; and (b) spectra at $t=(0,1,9)$. The evolution is qualitatively similar to that on the plane. 

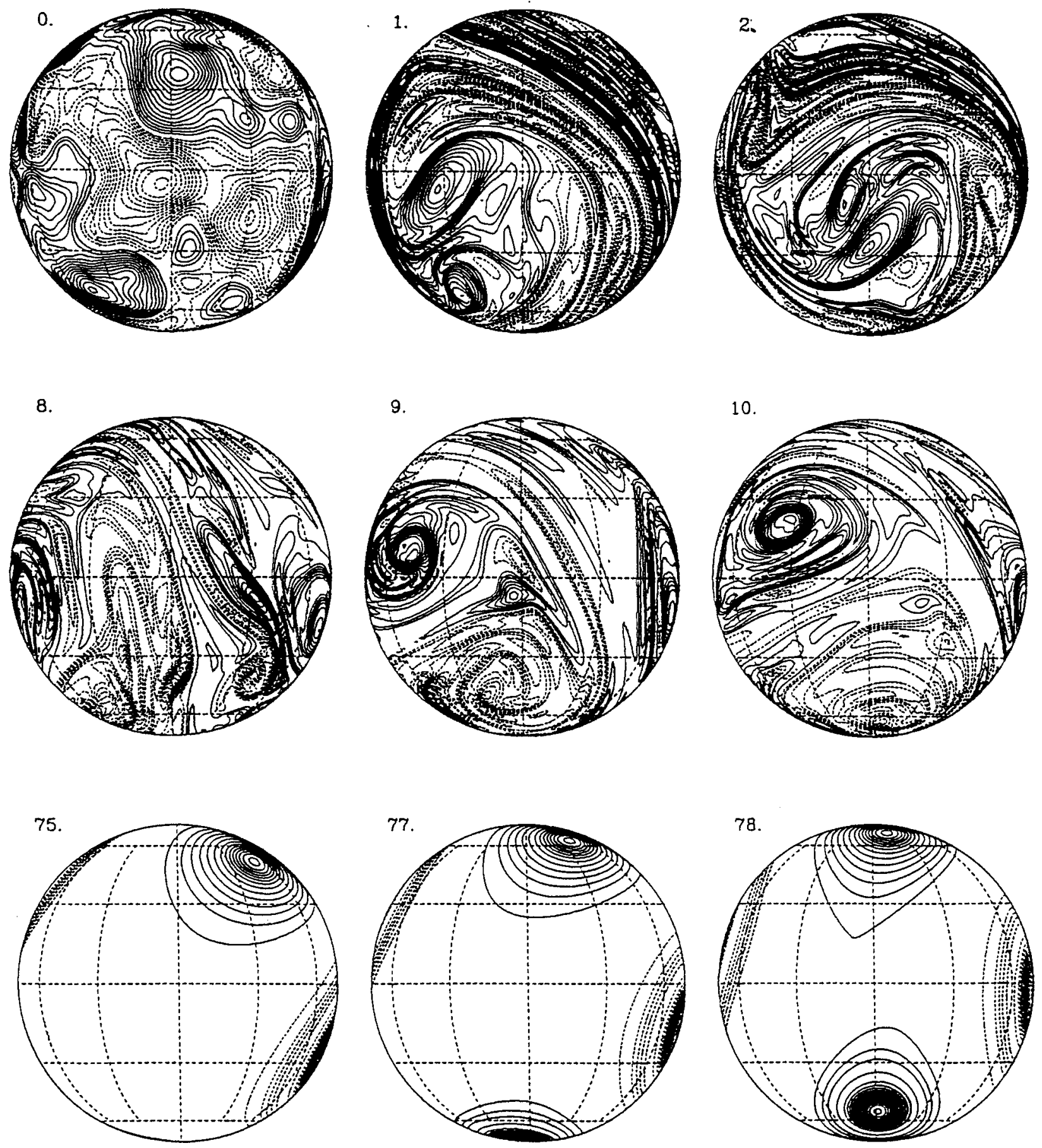

FIG. 3. Vortical quadrupole final-state configuration on a nonrotating $(R \rightarrow \infty)$ sphere, a direct consequence of angular momentum conservation. In this run, $t=1$ corresponds to approximately four vortex turnaround times. Solid (dashed) contours depict positive (negative) vorticity values.

but due to the initial peak location, which we now address.

Figure 7(b) shows the effect of varying $n_{0}=\{2,3,7,14\}$ with fixed $\left(p, \nu_{2 p}, \gamma\right)=\left(2,1.0 \times 10^{-8}, 20\right)$. As can be seen from Fig. 1(b), the variations in $n_{0}$ correspond to energy and enstrophy being primarily contained in large $\left(n_{0}=2\right)$ or small $\left(n_{0}=14\right)$ scales. It is clear from Fig. 7(b) that, depending on $n_{0}$, two distinct behaviors occur at the late stage (as defined above); the vortex-size distributions are also correspondingly different. For $n_{0}=\{7,14\}$ the inertial range is "kinked" upward, which is qualitatively similar to that re- ported by Yoden and Yamada, ${ }^{9}$ while for $n_{0}=2$ the kink is downward, similar to observations reported by Santangelo et al. ${ }^{5}$ and Dritschel. ${ }^{10}$ The $n_{0}=3$ case exhibits the "crossover" between the two types of behaviors; it is similar to the spectra in McWilliams ${ }^{1}$ and Benzi et al. ${ }^{4}$ Hence, there exists a clear monotonic relationship between $n_{0}$ and the kink direction.

From our study of the influence of initial spectra, several new conclusions can be drawn. First, at the current resolution, the spectral evolution is much more sensitive to the 


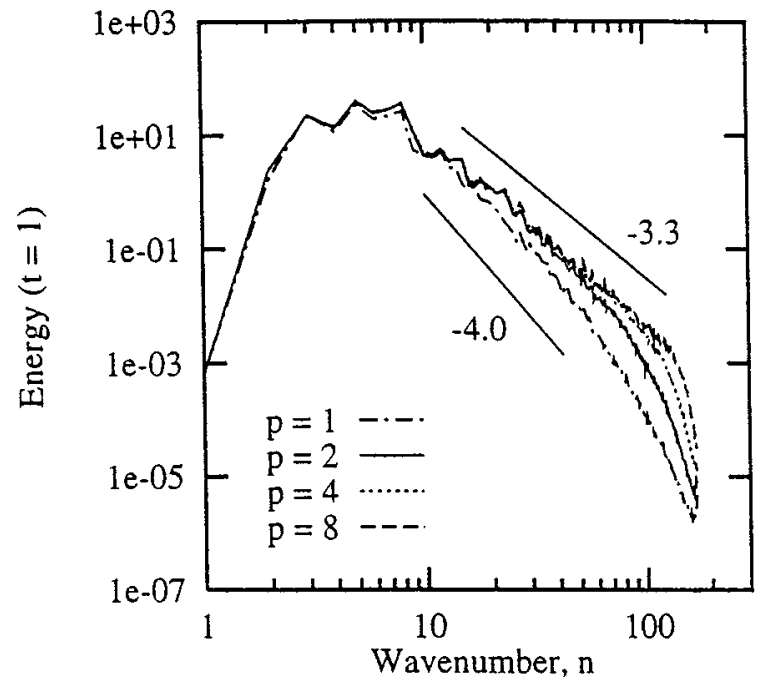

FIG. 4. Spectra at an early time, $t=1$, for runs A1-A4 (cf. Table II), for which $p=\{1,2,4,8\}$, respectively. The inertial range is broader and less steep with higher powered dissipation. The corresponding physical space picture is shown in Fig. 5.

initially most-energetic scale than to the spectral bandwidth. Since the question of time scale is ruled out by comparing the runs at the "well-decayed" phases of the evolution (i.e., when all the runs are well into the flat region of enstropy decay and the coherent structures have existed for many turnaround times), dissipation characteristics must play a significant role here. Second, the isolation of sensitivity to $n_{0}$ also suggests that the variety of previous results concerning the inertial range slope can be qualitatively understood in terms of the initial peak-energy scale used by the different investigations. And, third, the study also suggests that, in forced studies some of these sensitive, "free" features may be obscured or overwhelmed by the prescribed forcing, and that those studies might benefit from additional sensitivity tests, supplementing the works of Refs. 13 and 33.

In summary, our exploration of the simulation conditions leads us to conclude that while the general qualitative behavior (i.e., the emergence of long-lived coherent structures) is robust, many quantitative aspects such as the slope of the inertial range spectra and the population statistics of vortices and filaments in the flow are severely affected by the choice of dissipation and of the initial spectrum. Hence, our work suggests that the recent findings of self-similar universality ${ }^{34,4}$ might be put on firmer ground in a series of

TABLE II. The $p$ variation: $n_{0}=7, \gamma=20$, and T170 resolution. Here $p$ is the power of the hyperdissipation operator, $\nu$ is the viscosity coefficient, $x(1)$ is the inertial range slope at $t=1, \mathscr{E}$ is the energy, and $\mathscr{P}$ is the palinstrophy.

\begin{tabular}{cccccc}
\hline \hline Run & $p$ & $\nu_{2 p}$ & $x(1)$ & $\mathscr{E}(1) / \mathscr{E}(0)$ & $\mathscr{P}(1) / \mathscr{P}(0)$ \\
\hline A1 & 1 & $2.0 \times 10^{-4}$ & $-4.0 \pm 0.1$ & 0.768 & 0.254 \\
A2 & 2 & $1.0 \times 10^{-8}$ & $-3.8 \pm 0.1$ & 0.799 & 0.899 \\
A3 & 4 & $3.0 \times 10^{-17}$ & $-3.6 \pm 0.1$ & 0.950 & 1.76 \\
A4 & 8 & $3.0 \times 10^{-35}$ & $-3.3 \pm 0.1$ & 0.997 & 2.64 \\
\hline \hline
\end{tabular}

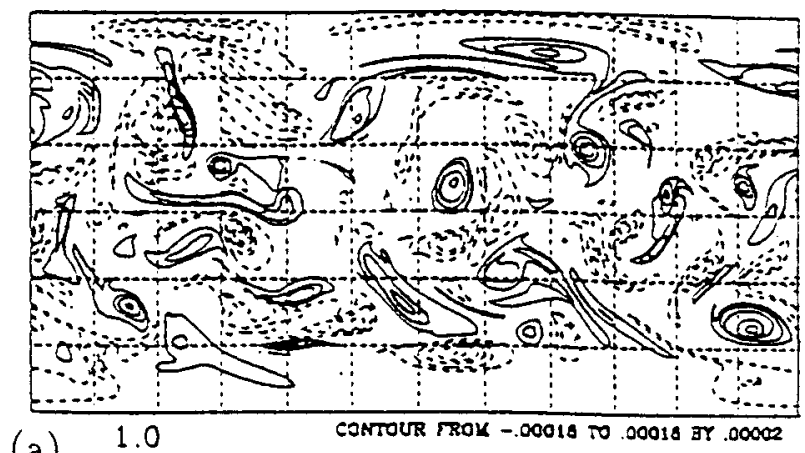

(a)
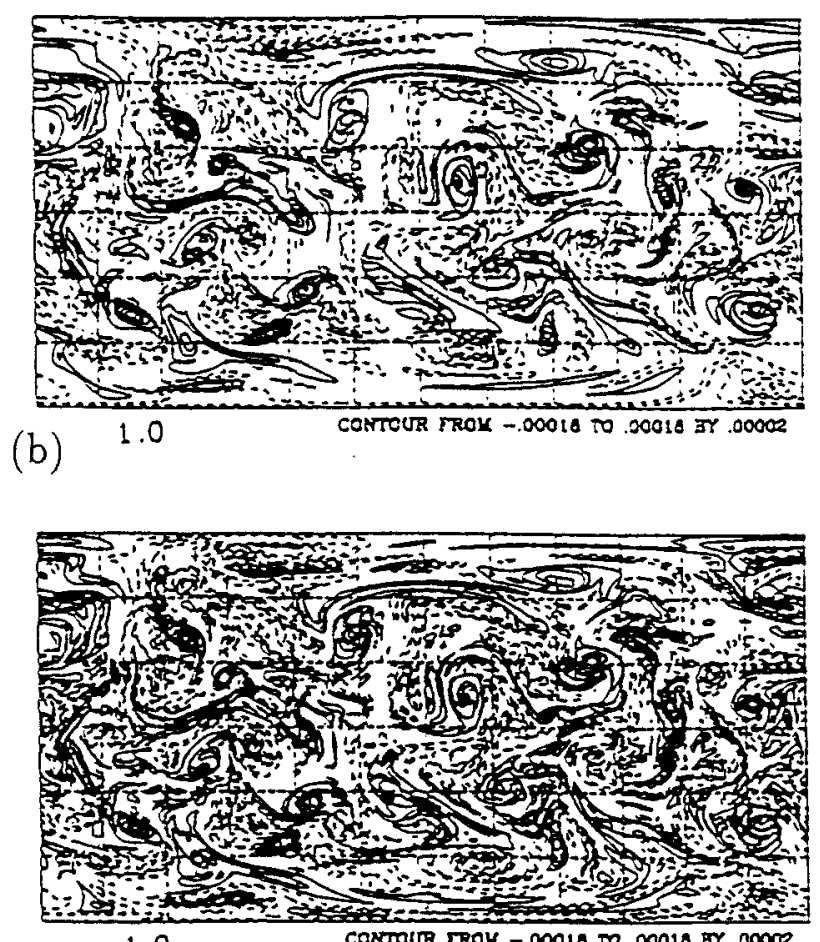

(c) CONTOUR TROK -.00018 TO .00018 BY .00002

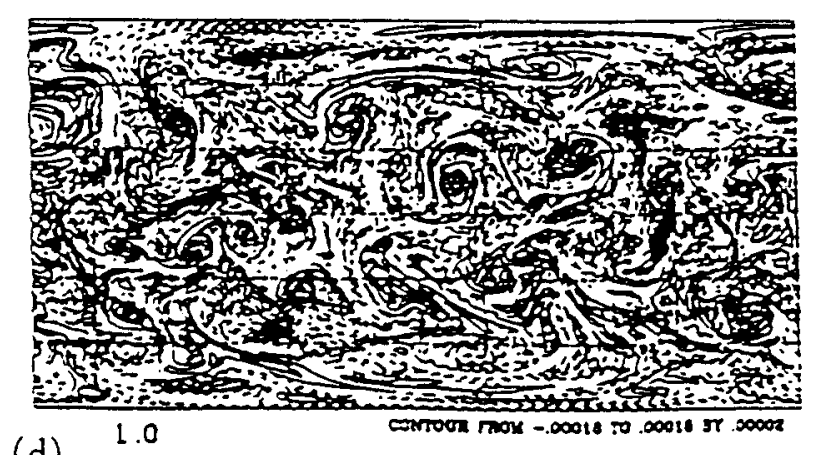

(d)

FIG. 5. The vorticity field in cylindrical-equidistant projection for runs A1-A4 [panels (a)-(d), respectively] at $t=1$. Solid (dashed) contours depict positive (negative) vorticity. The higher-powered dissipation admits larger quantity of space-filling filamentary structures.

calculations such as the one presented here, showing the robustness of that result to a variety of simulation conditions. On the other hand, the high sensitivity to simulation conditions and the well-known inadequacy of a purely spectral 

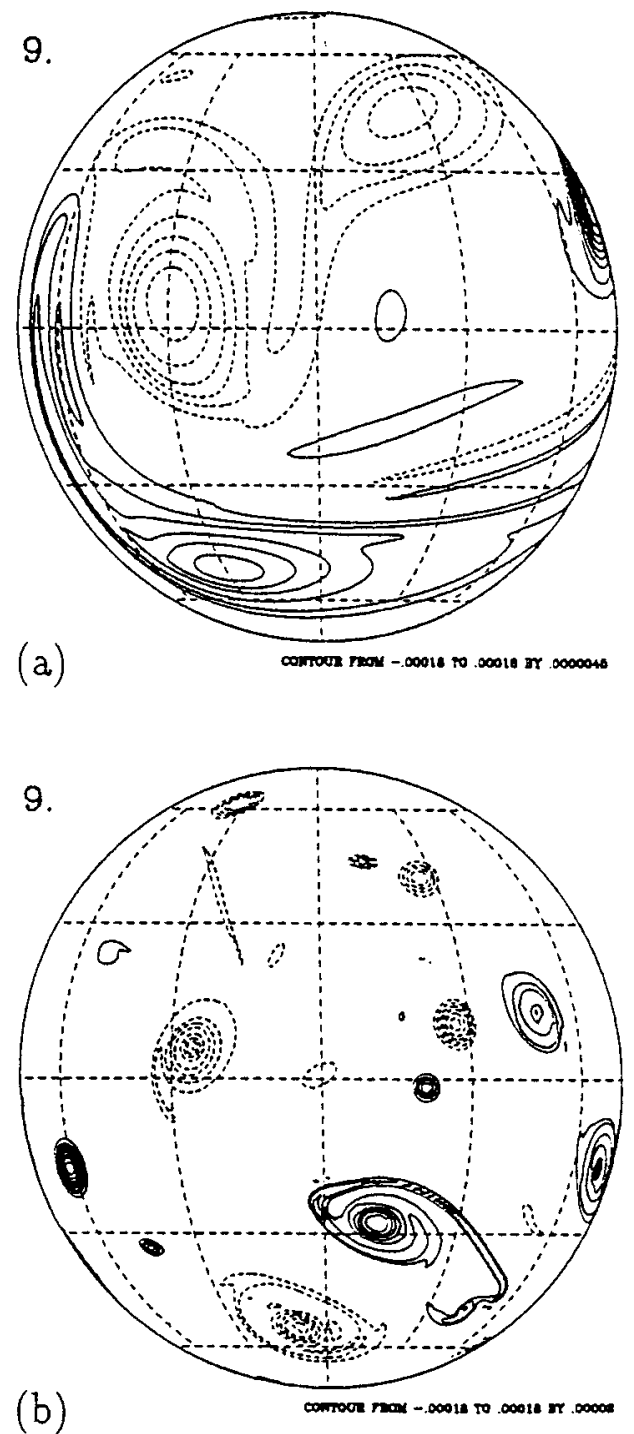

FIG. 6. The vorticity field for runs A1(a) and A4(b) at $t=9$ in orthographic projections. The contour levels are reduced in (a) to reflect the greater loss in energy. The large difference of vortex population in the two cases is due to dissipation.

description of turbulence lend support to the recent attempts to emphasize physical space diagnostics ${ }^{6,7,10}$ and justify our own emphasis on the robust physical space features to which we now turn our attention.

\section{ROTATION EFFECTS}

In this section, we move one step closer toward a geophysically realistic situation by introducing rotation into the purely 2-D system described by Eq. (12). The rotating, nondivergent spherical system (cf. Table I) with numerical dissipation is governed by the nondimensional dynamical equation:

$$
\partial_{t} \zeta+J\{\psi, \zeta\}+R^{-1} \partial_{\lambda} \psi=(-1)^{p+1} \nu_{2 p} \Delta^{p} \zeta,
$$

which we directly integrate in the runs described in this section; here, the curvature $a / L$ is fixed and hence ignored. It is
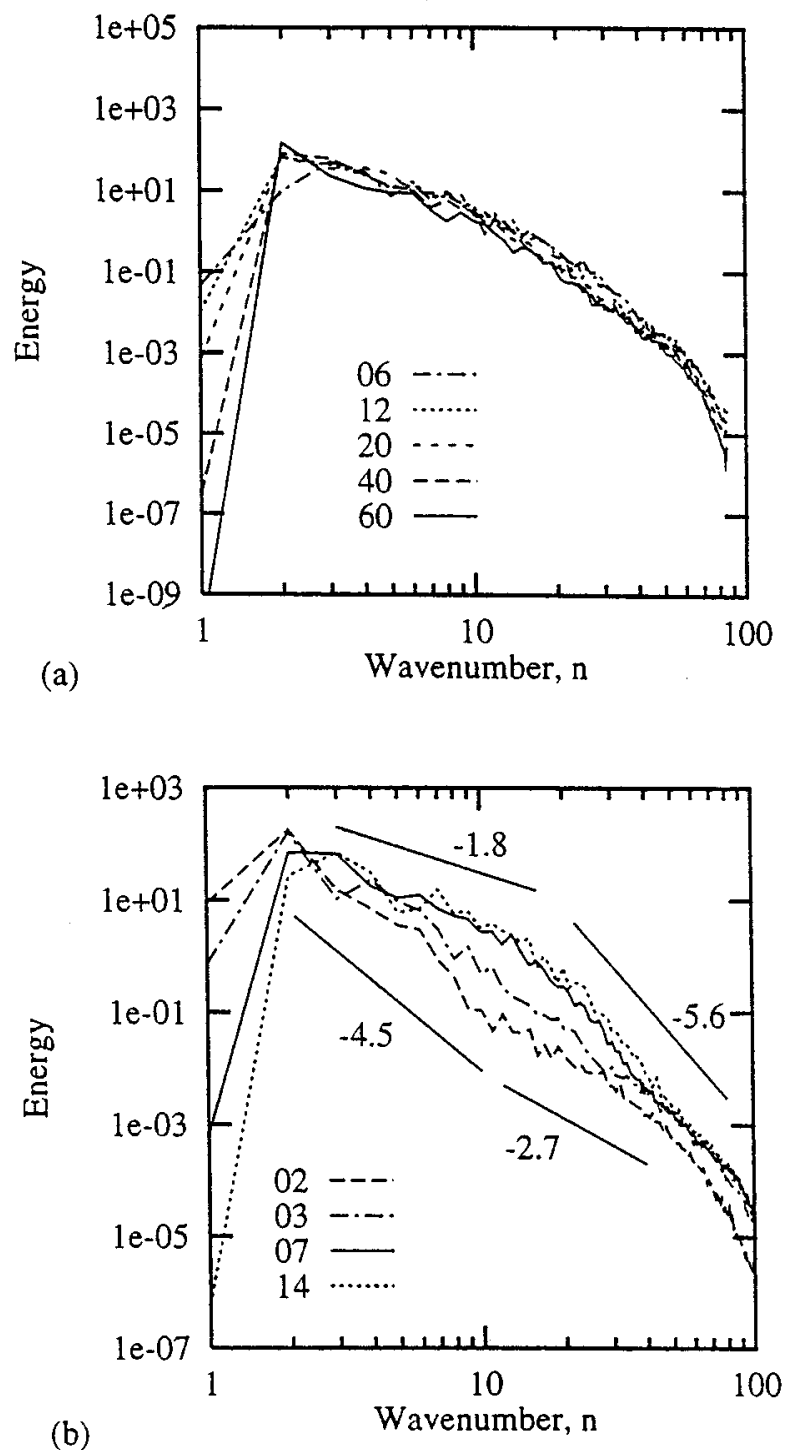

FIG. 7. Initial condition variations: spectra at $t=9$ for (a) $\gamma=\{6,12,20,40,60\}$ and (b) $n_{0}=\{2,3,7,14\}$. Long time evolution is not very sensitive to $\gamma$, but $n_{0}=2$ and $n_{0}=(7,14)$ leads to distinctive differences with "kinks" in the opposite direction. Here $n_{0}=3$ exhibits the "cross-over" between the two behaviors.

worth recalling that this equation is the fundamental statement of the material conservation of potential vorticity, $q \equiv \zeta_{a}$ (recall that $h$ is constant in this model) in the inviscid limit. Note also that the rotating sphere offers one important additional level of realism absent from the more

TABLE III. The $R$ variation: $n_{0}=7, \gamma=20, p=4, \nu_{8}=3.0 \times 10^{-17}$, and T85 resolution. Here $R$ is the Rossby number, $x(t)$ is the inertial range slopes at time $t, n_{\beta}$ is the equatorial $\beta$ based Rhines scale, and $\bar{n}$ is the energy centroid.

\begin{tabular}{cccccccccc}
\hline \hline Run & $R$ & $x(1)$ & $x(3)$ & $x(9)$ & $x(23)$ & $n_{\beta}$ & $n-(1)$ & $n-(9)$ & $n-(23)$ \\
\hline B1 & $\infty$ & -3.6 & -3.9 & -4.9 & -4.9 & $\ldots$ & 7.9 & 4.3 & 3.4 \\
B2 & 0.400 & -3.7 & -3.7 & -3.6 & -4.0 & 1.8 & 7.9 & 3.8 & 2.6 \\
B3 & 0.200 & -3.8 & -3.5 & -3.7 & -4.0 & 2.5 & 7.9 & 3.4 & 2.6 \\
B4 & 0.100 & -3.8 & -3.6 & -3.9 & -4.0 & 3.5 & 8.2 & 4.1 & 3.0 \\
B5 & 0.025 & -3.8 & -4.1 & -4.5 & -5.3 & 7.0 & 9.2 & 5.9 & 5.2 \\
B6 & 0.010 & -4.6 & -4.1 & -4.4 & -5.5 & 11. & 10.1 & 6.3 & 5.8 \\
\hline \hline
\end{tabular}


commonly used $\beta$ plane; namely, the $\beta$ (not only $f$ ) varies with latitude from the equator to the pole. This leads to interesting differences in the behavior between polar and equatorial regions, as discussed below.

The $\beta$-plane turbulence in a doubly periodic planar domain has been extensively studied. ${ }^{11,13,14}$ The constant parameter, $\beta$, absent in the nonrotating case, defines a new length scale, called the Rhines scale $L_{\beta} \equiv \pi \sqrt{2 U / \beta}$, at which the inverse energy cascade is strongly retarded by the anisotropy of the system - preventing the growth of vortices in the meridional direction. ${ }^{11}$ Thus, when forced, a steady, alternating field of elongated, zonal structures and jets is produced in $\beta$-plane turbulence. This mechanism has been proposed by Williams ${ }^{15}$ as a possible explanation for the observed, alternating zonal jet patterns in the atmosphere of Jupiter.
In non-Cartesian geometry, freely evolving $\beta$-plane turbulent flow has been studied by Marcus ${ }^{35}$ in annular geometry, and the first high resolution (T85) spherical simulations have recently been performed by Yoden and Yamada. ${ }^{9}$ Starting with initial conditions containing spatial symmetries and a spectrum, $E(n, 0) \propto n^{5} e^{-n / 2}$, Yoden and Yamada have found considerable differences with their and earlier, $\beta$-plane simulations. When $\Omega=0$, they detected a kinked inertial range with $x \sim-3$ for $n \leqslant 10$ and $x \sim-5$ for $n>10$. Also, when $\Omega$ was increased, they observed the flow field to be anisotropic (as in the plane) and reported the formation of an anticyclonic (westward) circumpolar vortex at high $\Omega$.

In this paper, we extend their results in three ways: longtime integrations are performed, no initial spatial symmetry is imposed, and more than one initial spectrum is considered.
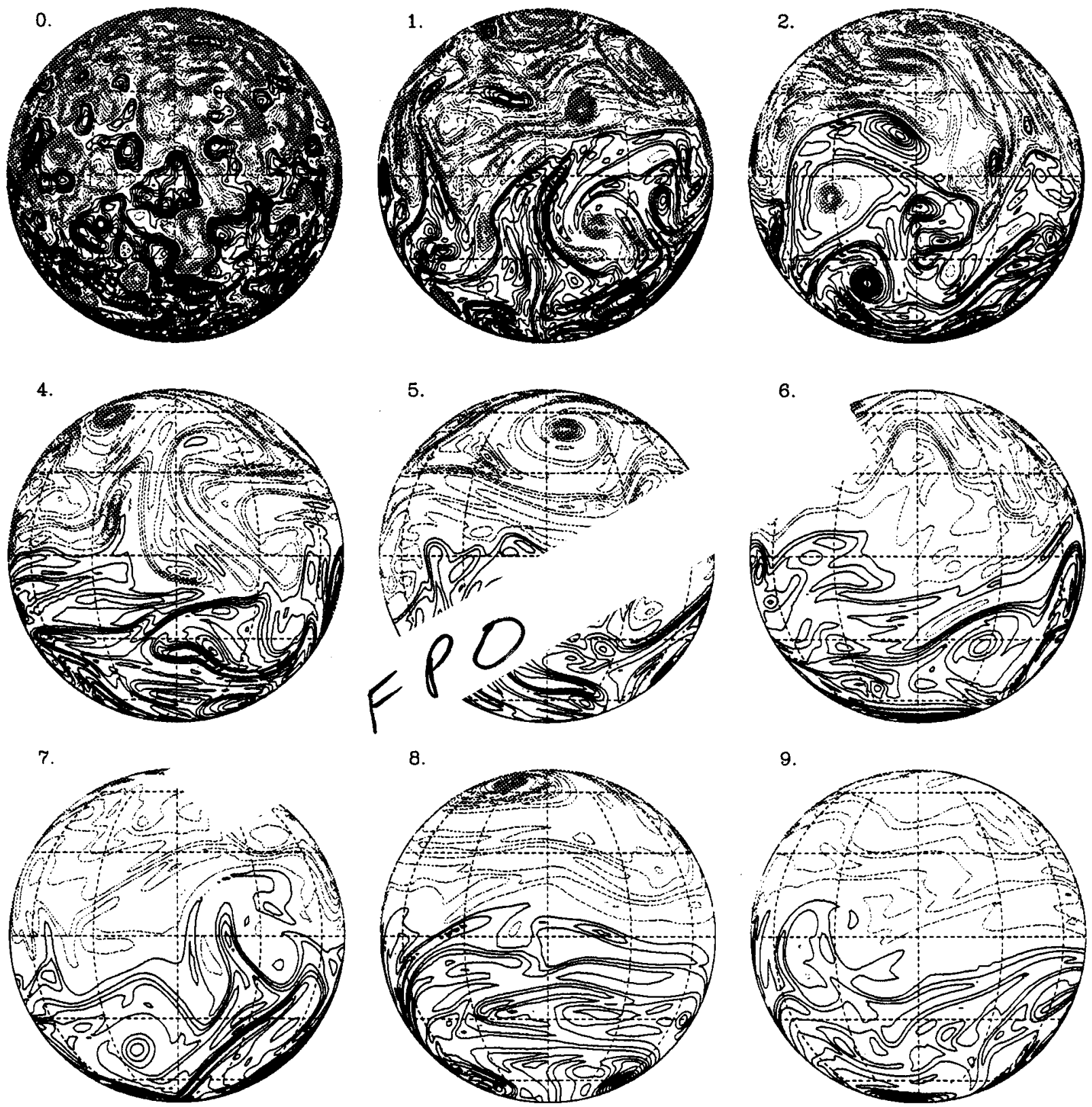

FIG. 8. Typical potential vorticity evolution under slow rotation $(R \sim 1)$ in the limit of $L_{D} \rightarrow \infty$ : run B3 of Table III in (a) orthographic projection centered at (longitude, latitude $)=(0,0)$, with positive (negative) values in red (blue), and (b) polar-stereographic projection at the North Pole. Here, $t=1$ corresponds to 2 rotations of the sphere, and $L_{\beta} / a=1$. 

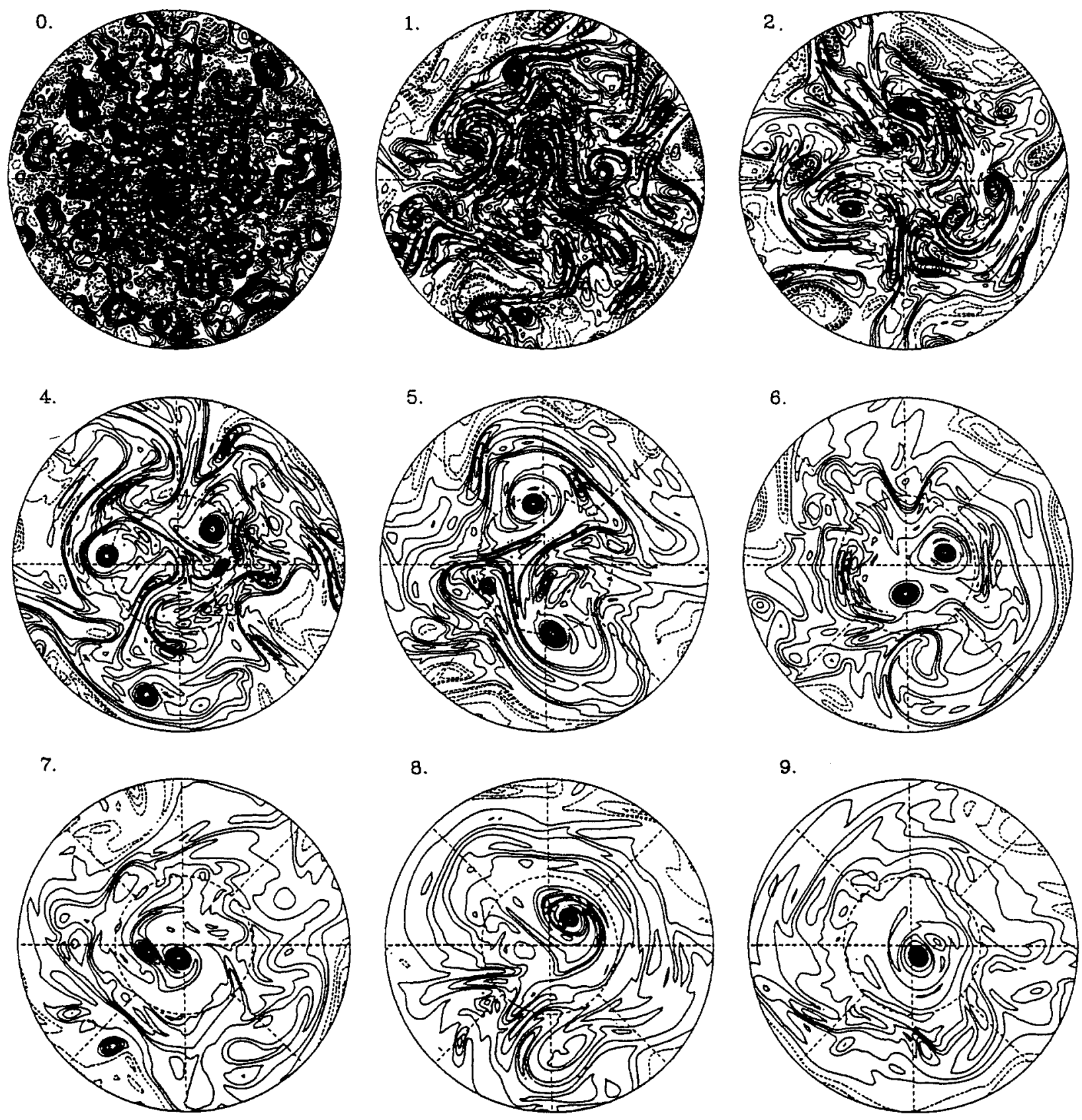

FIG. 8. (Continued.)

Table III lists one series of runs used for discussion in this section. The values of $\left(p, \nu_{2 p}, n_{0}, \gamma\right)=\left(4,3.0 \times 10^{-17}, 7,20\right)$ are fixed in these runs and only $\Omega$, or alternatively the Rossby number $R$, is varied. The listed runs (at T85 resolution) are chosen for their initial spectrum, which is sharply peaked about a scale smaller than $L_{\beta}$, allowing the flow field to undergo a significant amount of spectral broadening and inverse cascade. ${ }^{36}$ In all the runs, long-time integrations were carried out until flat enstrophy decay behavior was observed. We start by mentioning briefly some results regarding longtime evolution of the spectrum before presenting in detail the physical evolution and how it is affected by rotation.

The first result to report is that, as rotation increases, the inertial range slope at early times becomes steeper [see the $x$ (1) column in Table III]. This monotonic behavior of the slope $x$ with $\Omega$ for short integration time was previously reported by Yoden and Yamada ${ }^{9}$ and is due to the retardation of inverse cascade by the $\beta$ effect. However, as can be seen from the slopes at later times (cf. Table III), that behavior is not robust.

The energy centroid,

$$
{ }^{-}-(t) \equiv \frac{\sum_{n=1}^{n_{t}} n E(n, t)}{\sum_{n=1}^{n_{t}} E(n, t)},
$$

with $\bar{n}(0)=11.9$ (corresponding to $n_{0}=7$ ) is tabulated for several times in Table III; it provides a quantifying measure of the inverse energy cascade arrest. At the early stages, the inverse cascade is indeed retarded to a greater extent by a 
larger $\Omega$ (smaller $R$ ), leading to steeper spectra as in Ref. 9 [cf. the $\bar{n}(1)$ column in Table III]. However, this monotonic behavior with $\Omega$ is destroyed at later times when the flow field contains highly intermittent vortical structures [cf. the $\bar{n}(9)$ and $\bar{n}(23)$ columns in Table III]. The vorticity kurtosis, $\mathrm{Ku}_{\zeta}(t) \equiv\left\langle\zeta^{4}\right\rangle /\left\langle\zeta^{2}\right\rangle^{2}$ (used to quantify the intermittency), at $t=9$ for run B1 is 18 , approximately three times larger than for the rest of the runs. On the other hand, we have found that the monotonic behavior can be sustained for longer periods, using an initial spectrum with larger $n_{0}$. It should be clear at this point that many spectral behaviors are possible; and, the result again points to the danger of making quanti- tative comparisons of spectral slopes, be they against other spherical results or against planar results, especially if only a single simulation condition is considered.

The general physical space behavior, fortunately, can be considerably more robust. That is, from our many simulations, we have found that the resolution and $n_{0}$ variations do not qualitatively affect the features discussed below. The key physical results of this section is that the rotation $\Omega$ produces two qualitatively different physical behaviors: one (at small $\Omega$ ) in which there is a substantial latitudinal motion by the growing vortices, and the other (at large $\Omega$ ) in which structures are initially latitudinally confined but quickly give way
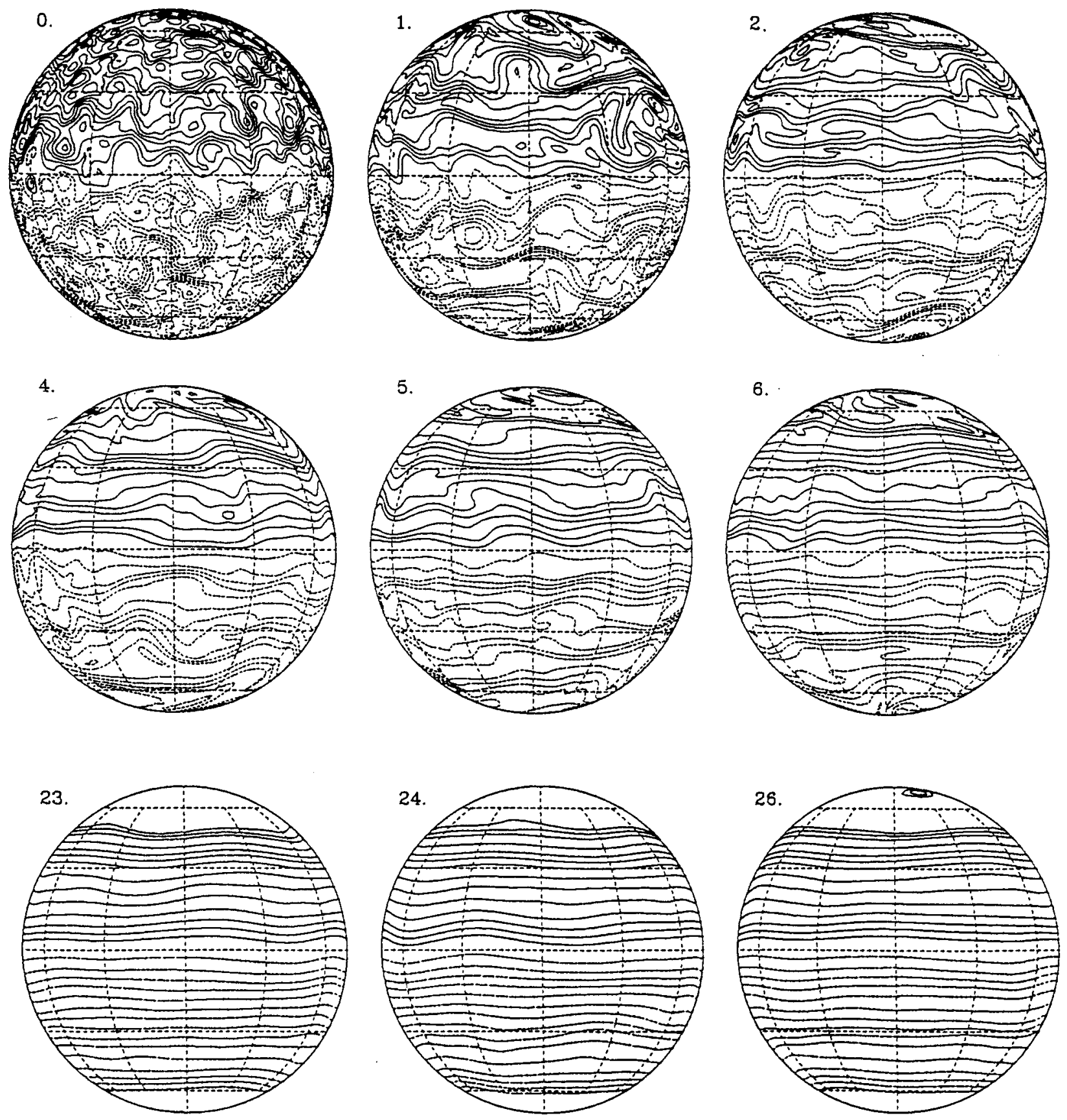

FIG. 9. Typical potential vorticity evolution under rapid rotation $\left(R \ll 1\right.$ and $\left.L_{D} \rightarrow \infty\right)$ : run B5 of Table III. The views are as in Fig. 8 . The $L_{\beta}$ scale is shown in the last frame in (b). Notice the formation of the anticyclonic, circumpolar vortex. Here, $t=1$ corresponds to 16 rotations of the sphere, and $L_{\beta} / a=0.3$. 

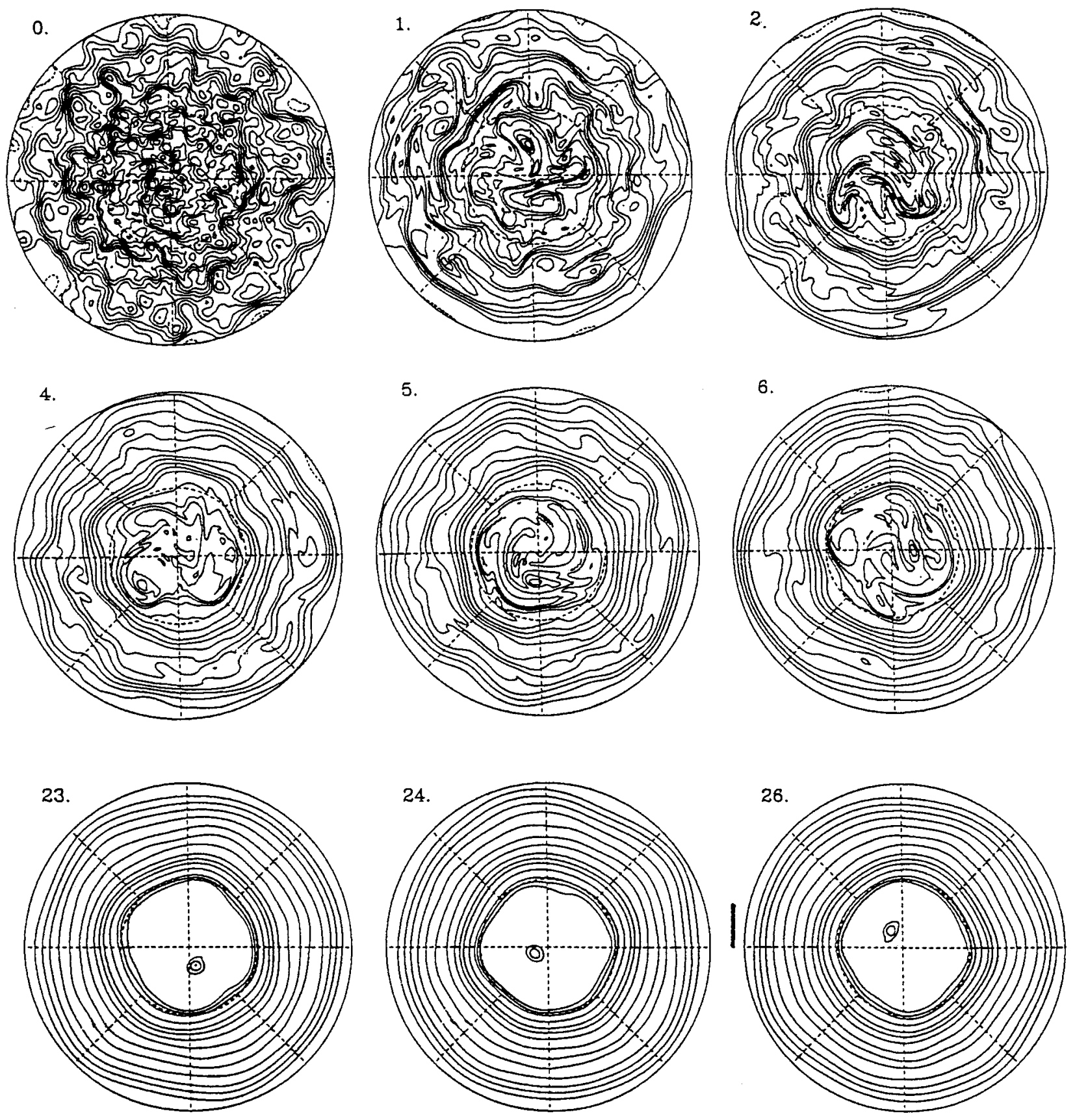

FIG. 9. (Continued.)

to a field dominated by a pair of circumpolar vortices. We now give an illustration of each of these two behaviors.

First, Fig. 8 shows an orthographic (a) and a polarstereographic (b) view of the potential vorticity evolution in a typical "low-rotation" $(R \sim 1)$ case, specifically run B3 in Table III; in this figure, $t=1$ corresponds to approximately 2 rotations of the sphere and $L_{\beta} / a=1$, where the equatorial value of $\beta$ has been used since the value of $\beta$ is variable. In this case, the effect of spatially varying $\beta$ on the sphere can be clearly seen. In the early stages, the latitudinal variation of $\beta$ separates the field, roughly speaking, into two dynamical regions [see, for example, the $t=5$ frame of Fig. 8(a)]: a low-latitude region populated with mostly elongated structures and large-scale waves and a high-latitude region con- taining well-defined coherent vortices undergoing continuous mergers in the poleward direction; the mergers are clearly seen in the polar view of Fig. 8(b). These figures highlight the fact that the rotating sphere is really a composite environment, $\beta$-plane-like at low latitudes and $f$-plane-like near the poles, and exhibits a much more complex physical space behavior than the simpler $\beta$ plane.

Furthermore, Fig. 8(a) clearly illustrates the long-time relaxation of $q$ toward a geostrophic, solid-body rotation state. ${ }^{37}$ Notice how, starting from a field containing both positive (red) and negative (blue) values of $q$ in both hemispheres at $t=0$, the flow clearly separates via selforganization into a northern hemisphere containing strictly positive values of $q$ and a southern hemisphere containing 
only negative values of $q$ at $t=8$. We note that the relaxation is not due to dissipation, since only $4 \%$ of the initial energy is lost at the end of the run and is mainly due to the inversely cascading turbulent evolution. To the best of our knowledge, this type of evolution of $q$ toward $f$ from random initial conditions has not been demonstrated in previous simulations. $^{38}$

Second, Figs. 9(a) and 9(b) illustrate a typical "highrotation" $(R \ll 1)$ case, specifically run B5 in Table III. In this example, $t=1$ corresponds to approximately 16 rotations of the sphere and $L_{\beta} / a=0.3$. The distinctive behavior at high rotation is the formation of anticyclonic circumpolar vortices, as previously reported by Yoden and Yamada. ${ }^{9}$ In our computations, in which no initial spatial symmetries have been used, we have found that polar vortices generally form at both poles-as might be expected. Moreover, when the polar vortices, bounded by large $q$ gradients, are fully formed, $q$ is well homogenized in their interiors-but not elsewhere (see the last three frames of Fig. 9), a feature not obvious in Ref. 9 since the $\psi$ field is plotted rather than the $q$ field (see Fig. 7 in Ref. 9).

Unlike in the forced planar cases, ${ }^{11,13}$ zonal jet structures are not particularly well defined in our rotating, nondivergent decaying experiments. This is due to a combination of the spatially varying $\beta$, the anisotropic energy arrest in spectral space, and the decaying nature of unforced evolution. It would seem that steady jets in the pure 2-D case require a continuous supply of enstrophy for sustenance. Table III also shows that, at the stage when the inverse cascade has nearly halted ( $t=23), \bar{n}<n_{\beta}$ at high rotation rates, as was found in the forced $\beta$-plane experiments of Vallis and Maltrud. ${ }^{14}$ Therefore, although the meridional alternations do generally increase in number for greater values of $\Omega$ initially [see the $n^{-}$(23) column of Table III], the Rhines scale $L_{\beta}$ does not appear in this case to be a viable measure of the number of bands on the sphere, and ultimately not even in a qualitative sense, since the jets do not last. This is particularly clear in physical space, where the initially large number of alternations quickly gives way to a field dominated by strong polar vortices, and the $q$ contours are nearly uniform in the meridional direction (cf. Fig. 9). In this situation, jets are barely present and difficult to detect, except at the edges of the polar vortices.

Finally, in our series of many nondivergent runs under varying rotating conditions, no asymmetry between cyclonic and anticyclonic ${ }^{39}$ vorticity is observed. The cyclone/ anticyclone asymmetry can be quantified and tracked via the skewness of the vorticity field, defined by

$$
\mathrm{Sk}_{\zeta}(t) \equiv \frac{\left\langle\zeta^{3}\right\rangle}{\left\langle\zeta^{2}\right\rangle^{3 / 2}}
$$

Figure 10(a) shows the vorticity skewness time series for run B4 in Table III, a typical high rotation case. The small nonzero values are due to the numerical discretization. A complimentary quantity, confirming the absence of vorticity asymmetry in these nondivergent rotating cases, is the corresponding vorticity pdf, shown in Fig. 10(b) for $t=4$. Note the approximately equal areas in the left and right wings, corroborating the zero skewness. Therefore, our computa-
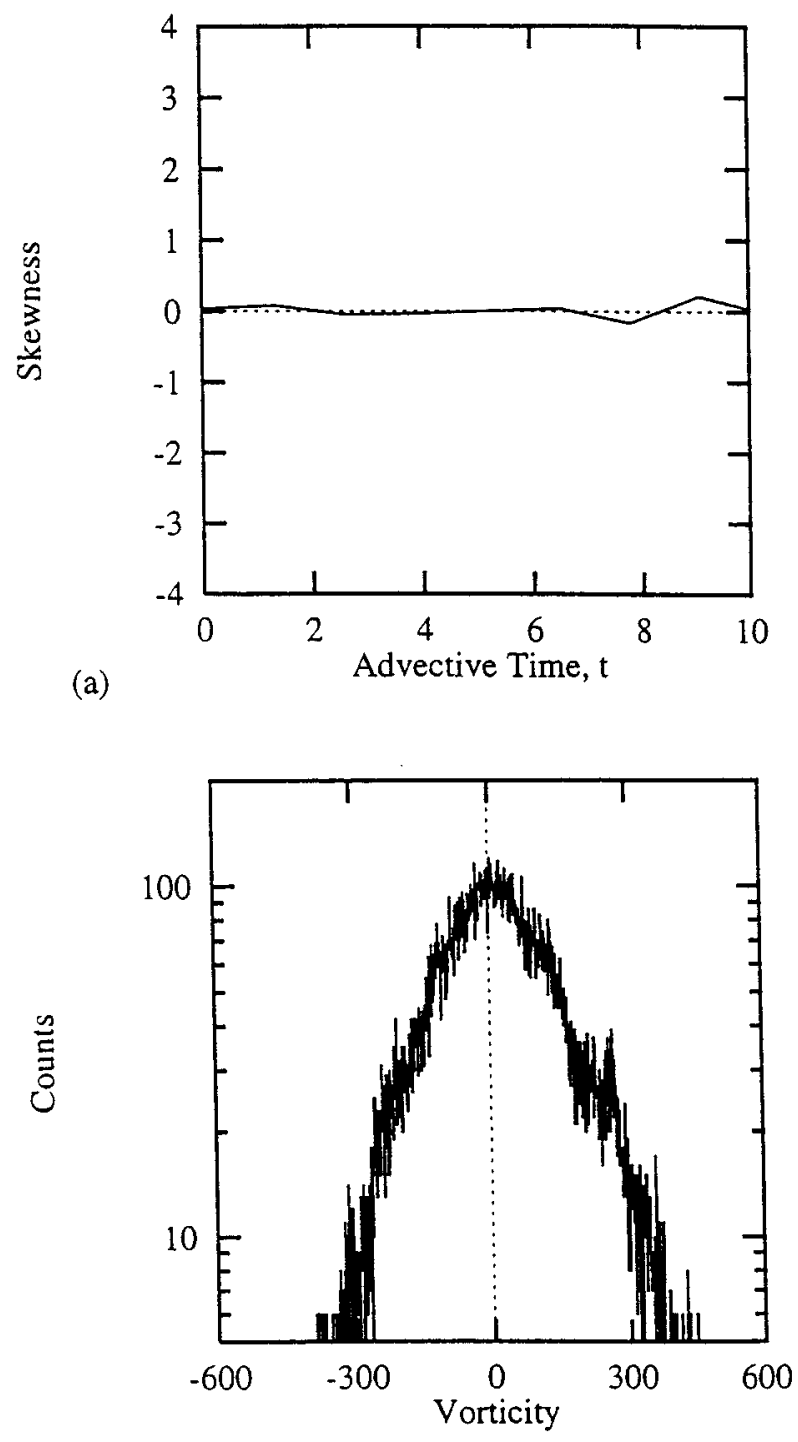

(b)

FIG. 10. Absence of cyclone/anticyclone asymmetry in the nondivergent, rotating case: (a) the vorticity skewness time series for run B4 of Table III and (b) the pdf of the vorticity field at $t=4$.

tions provide evidence that the $\beta$ effect alone is clearly not sufficient to explain the predominance of anticyclonic vortices in the atmospheres of the giant planets. ${ }^{40}$ It is through the addition of one further physical complexity, specifically the presence of free surface, that asymmetry between cyclones and anticyclones is found to appear. The effects of a free surface is the subject of the next section.

\section{DEFORMATION RADIUS EFFECTS}

In this section, we further increase the physical complexity by considering a rotating fluid bounded by a free surface; this allows for horizontal divergence of the velocity field and hence vortex-tube stretching in the flow. For this, the full set of shallow-water equations (SWE) must be used. The spherical SWE in nondimensional vorticity-divergence form, as they are used in the numerical integration procedure, are 


$$
\begin{aligned}
\partial_{t} \zeta_{a}= & -\frac{1}{\widetilde{a}\left(1-\mu^{2}\right)} \partial_{\lambda}\left(\widetilde{U} \zeta_{a}\right)-\frac{1}{\widetilde{a}} \partial_{\mu}\left(\widetilde{V} \zeta_{a}\right)+\hat{\mathscr{D}} \zeta \\
\partial_{t} \delta= & \frac{1}{\widetilde{a}\left(1-\mu^{2}\right)} \partial_{\lambda}\left(\widetilde{V} \zeta_{a}\right)-\frac{1}{\widetilde{a}} \partial_{\mu}\left(\widetilde{U} \zeta_{a}\right) \\
& -\Delta\left(F^{-2}\left(1+h^{\prime}\right)+\frac{\widetilde{U}^{2}+\widetilde{V}^{2}}{2\left(1-\mu^{2}\right)}\right)+\hat{\mathscr{D}} \delta
\end{aligned}
$$$$
-\delta+\hat{\mathscr{D}} h^{\prime},
$$

$$
\partial_{t} h^{\prime}=-\frac{1}{\widetilde{a}\left(1-\mu^{2}\right)} \partial_{\lambda}\left[\widetilde{U}\left(1+h^{\prime}\right)\right]-\frac{1}{\widetilde{a}} \partial_{\mu}\left[\widetilde{V}\left(1+h^{\prime}\right)\right]
$$

where

$$
\begin{aligned}
& \zeta_{a} \equiv \frac{1}{\widetilde{a}\left(1-\mu^{2}\right)} \partial_{\lambda} \widetilde{V}-\frac{1}{\widetilde{a}} \partial_{\mu} \widetilde{U}+R^{-1} \mu, \\
& \delta \equiv \frac{1}{\widetilde{a}\left(1-\mu^{2}\right)} \partial_{\lambda} \widetilde{U}+\frac{1}{\widetilde{a}} \partial_{\mu} \widetilde{V},
\end{aligned}
$$
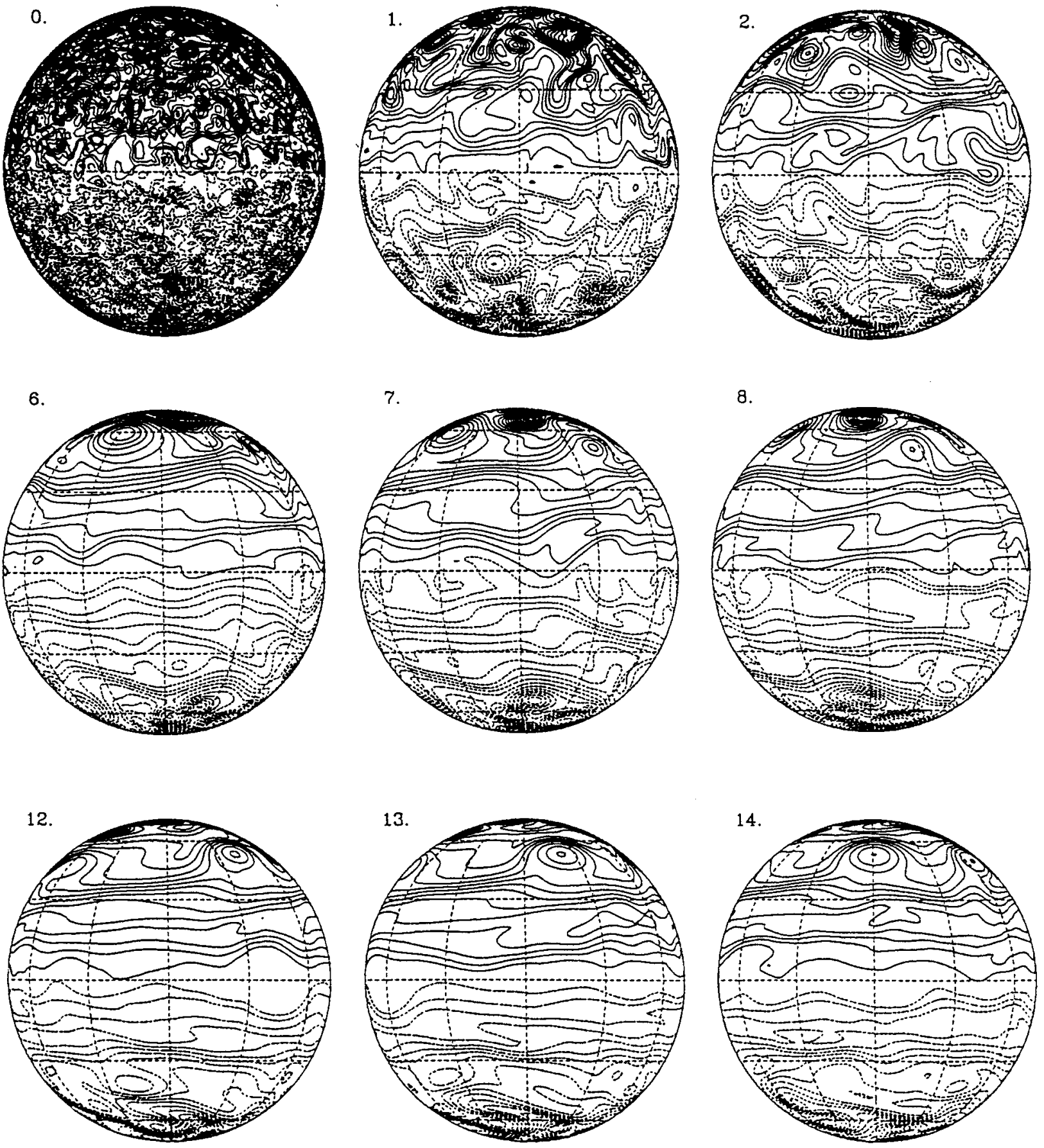

FIG. 11. Typical potential vorticity evolution of a run with finite deformation radius ( $\left.L_{D} / a=0.03\right)$ in (a) orthographic and (b) polar-stereographic views. The field does not condense into a polar vortex and bands are formed. Here, $t=1$ corresponds to approximately 20 rotations of the sphere and $L_{\beta} / a=0.3$. The $L_{\beta}$ scale is shown in the last frame of (b); the $L_{D}$ scale is too small to be shown. 

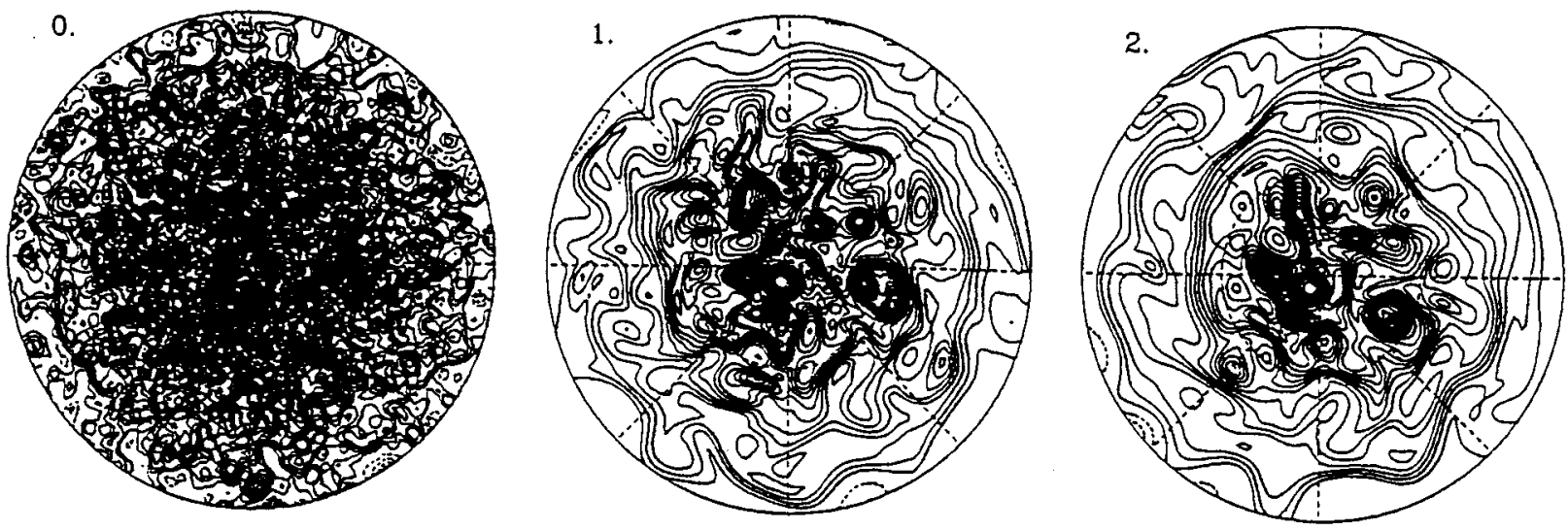

6.
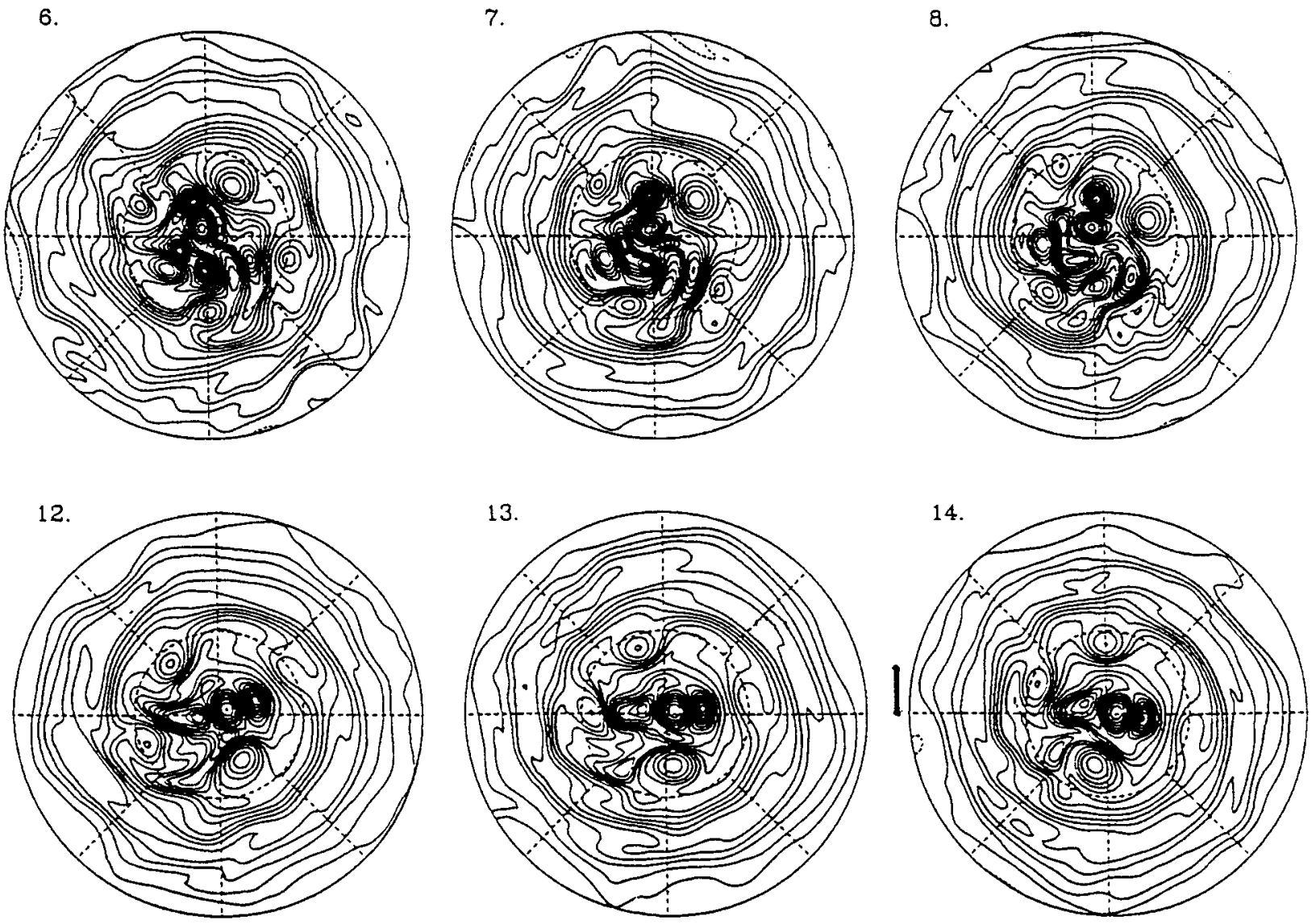

FIG. 11. (Continued.)

and $h^{\prime}$ are the three prognostic variables, representing absolute vorticity, velocity divergence, and surface deviation, respectively, and

$$
\begin{aligned}
& \widetilde{U} \equiv u^{\prime} \sqrt{1-\mu^{2}}, \\
& V^{\sim} \equiv v^{\prime} \sqrt{1-\mu^{2}},
\end{aligned}
$$

with $\mu \equiv \sin \vartheta$ and $\tilde{a} \equiv a / L$. Here $\hat{\mathscr{D}}$ is the hyperdissipation operator, $(-1)^{p+1} \nu_{2 p} \Delta^{p}$. We remind the reader that the potential vorticity, $q$, is now equal to $\zeta_{a} / h$.

Whereas in the previous section the only physical length scale in the problem was $L_{\beta}$, the presence of a free surface introduces here a new important length scale in the physical system, $L_{D}=\sqrt{g H} / 2 \Omega$. The nondivergent systems we have considered in the previous two sections correspond to the limit $L_{D} \rightarrow \infty$, whereas it is finite in this system.

In the asymptotic limit of $R \ll 1$ and $F \ll 1$, where the $S W E$ reduce to the so-called "equivalent barotropic" equations on the $f$ plane, the effect of a finite $L_{D}$ on the vorticity dynamics of a shallow rotating fluid have been studied in some detail. ${ }^{41-43}$ In this case, it is well known that the vortex interaction decays exponentially with separation over an $e$-folding scale comparable to $L_{D}$, in contrast with the nondivergent case where the interaction is long range and logarithmic. The shorter range implies that when vortices are of a size that is large compared to $L_{D}$ they are less coherent since one side of the vortex does not "see" the other side; hence, 


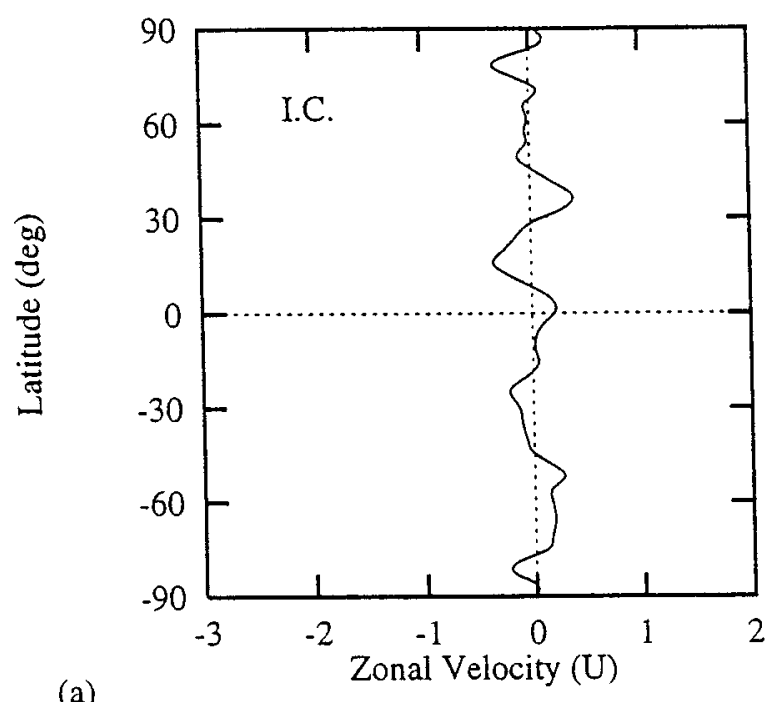

(a)

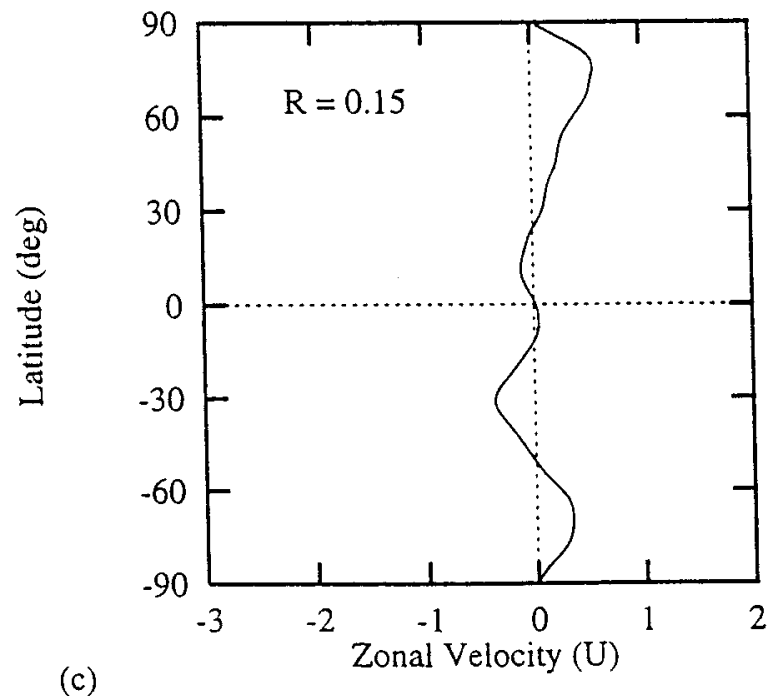

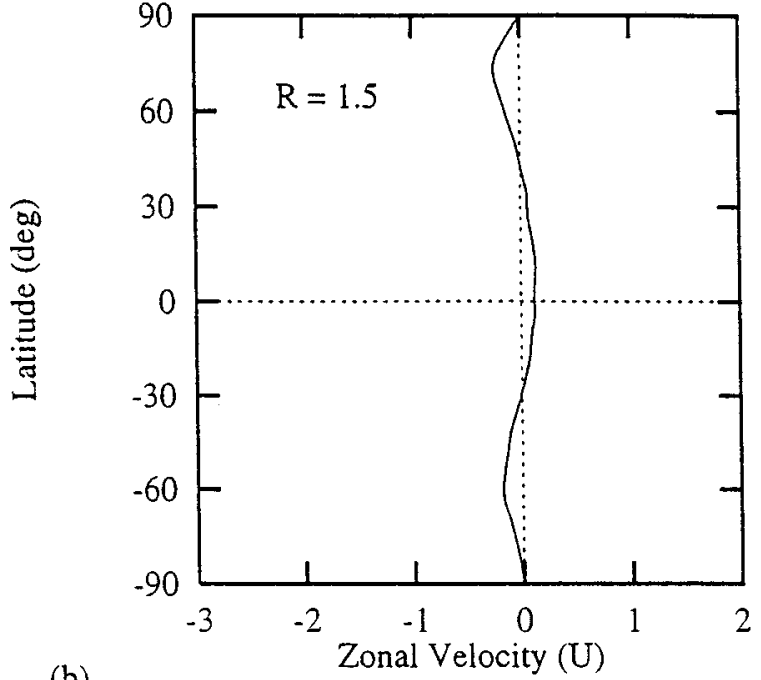

(b)

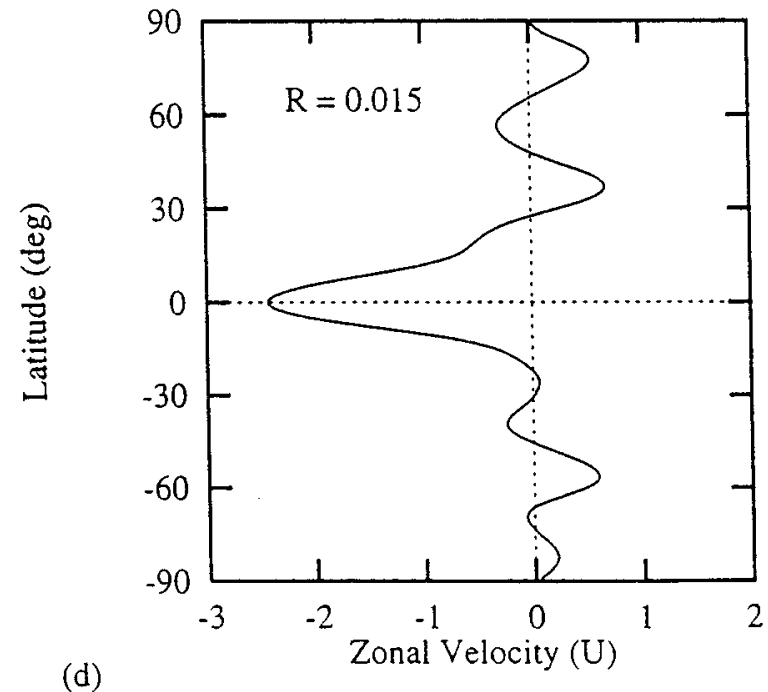

FIG. 12. The zonally averaged, zonal velocity in units of characteristic velocity as a function of latitude for several rotation rates: (a) the common initial profile and (b)-(d) the long-time, steady profiles for runs with $R=(1.5,0.15,0.015)$, respectively. All other parameters are identical: $\left(p, \nu_{2 p}, n_{0}, \gamma\right)=\left(3,4.0 \times 10^{-10}, 7,18\right)$. The number of jets increase with $\Omega$, as $R$ becomes small.

for $L \gg L_{D}$ vortices are found to be "blobby" and nonaxisymmetric. ${ }^{41-43}$

As for the full SWE (i.e., in nonasymptotic parameter regimes), the evolution of coherent structures has only recently been studied in doubly periodic, planar geometry. ${ }^{17-19}$ The key results are that the emergence and the eventual size of coherent structures are strongly influenced by the value of the deformation radius (in our case, $L_{D}$ provides a second, apparently crucial, inverse-cascade arrest mechanism). Furthermore, when the free surface variations are not small compared to the mean thickness of the fluid layer [i.e., $h^{\prime}=\mathscr{Q}(1)$ such that $\left.R B^{-1} \geqslant 0.13 \pm 0.04\right]$ a strong asymmetry develops between cyclonic and anticyclonic vortices, favoring the latter. $^{19}$

Since the full SWE admit gravity waves, it is necessary to initialize the flow with a balancing procedure. ${ }^{44}$ In this work, a bounded derivative method ${ }^{45}$ has been used to balance the initial condition. The bounded derivative method is based on the observation that a solution of the scaled equations with a time scale of $\mathscr{O}(1)$ must have a sequence of time derivatives of the dependent variables, also of $\mathscr{Q}(1)$. Thus, terms in SWE that contribute to large time derivatives are identified by performing a scaling analysis and are constrained to be on the same order as the slower, Rossby wave time scale. In theory, the higher the order of the constrained time derivative, the smoother the temporal evolution of the flow. In practice, we have found a second-order time and first-order $(R)$ bound to be adequate for this study.

We start by illustrating the key result of this section in Fig. 11: in the presence of a finite deformation radius, the potential vorticity does not condense into a polar vortex but takes the form of banded zonal structures-even when $\Omega$ is large. Contrast the evolution in Figs. 11(a) and 11(b) with the corresponding nondivergent case in Fig. 9. The runs in both figures have $L_{\beta} / a=0.3$; however, in Fig. $11 L_{D} / a=0.03$, whereas it is infinity in Fig. 9. The formation of banded 
structures leading to robust zonal jets is a general behavior that we have found common to all the runs, with $L_{D} / a<\frac{1}{3}$ in our many simulations. For larger values of $L_{D} / a$, the effect of the free surface is negligible and the evolution resembles the nondivergent or nonrotating cases of the previous sections; we have spanned the values of $L_{D} / a$ from 0.01 to 725 .

Notice further how, when $L_{D}$ is finite, the zonal structures are somewhat spiraling out of the pole and are made of regions of steep gradients separating well-homogenized bands of potential vorticity [see, for instance, frame $t=8$ in Fig. 11(b)]. The polar regions, of course, behave more like an $f$ plane, and hence vortices are not sheared away by the $\beta$ effect; at the same time, note how the unsheared vortices do not condense into a polar vortex, owing to the small $L_{D}$ halting the inverse cascade. It is well worth emphasizing here that the evolution of the flow is completely unforced; to the best of our knowledge, all previous studies that have produced steady banded structures have done so under forced conditions. $^{15,13,14} \mathrm{We}$ are demonstrating here for the first time that a freely evolving flow spontaneously self-organizes into such banded structures, provided the deformation radius is finite and the rotation is high.

The second result is a direct consequence of the fact that a finite deformation radius yields banded potential vorticity structures: one can directly observe in physical space how the number of robust jets (the bunching of $q$ contours) increases as the rotation rate is increased-even after a long time. In Fig. 12 we demonstrate this by plotting zonally averaged zonal velocity $\left[\bar{u} \equiv 1 / 2 \pi \int_{0}^{2 \pi} u(\lambda, \vartheta) d \lambda\right]$ profiles. Figure $12(\mathrm{a})$ is the common, random initial condition, while Figs. 12(b)-12(d) show the zonal profiles at long times for three different value of Rossby numbers, $R=1.5,0.15$, and 0.015 , respectively. After $t=23$, the profiles are perfectly steady for all three cases. It is also clear that, in our simulations with well-resolved and well-separated $L_{\beta}$ and $L_{D}$ scales, the former scale controls the jet scale and no secondary spectral peak in the vicinity of the latter scale is observed.

Although the number of zonal jets clearly increases with rotation rate, as predicted by Rhines, ${ }^{11}$ again we have been unable to quantitatively use the Rhine scale as a predictor of that number, showing that this behavior is not a result of forcing. In Fig. 12, the rough expected number of bands using the Rhines scale $\left(\pi a / L_{\beta}\right)$ is 1,4 , and 12 in panels (b), (c), and (d), respectively. As in Ref. 14, we suspect the spatial variation of $\beta$ to play an important role in moderating the number of bands and jets. We also point out that, unlike in the forced $\beta$-plane simulations, our spontaneously formed bands on the sphere are not perfectly zonal; they spiral at high latitudes. Therefore, the zonal averaging procedure destroys some of the (banded) features, which are otherwise more obvious in the full physical field (cf. Fig. 11).

A third interesting result can be obtained by varying the mean thickness, $H$ (hence the $L_{D}$ ), at a fixed rotation rate. This is illustrated in Fig. 13, where the zonally averaged velocities at $t=32$ for four runs with $L_{D} / a=(0.30,0.18,0.12,0.08)$ are shown; all other parameters are fixed. The point here is that a decreasing deformation radius yields an increasing equatorial jet; or, equivalently,

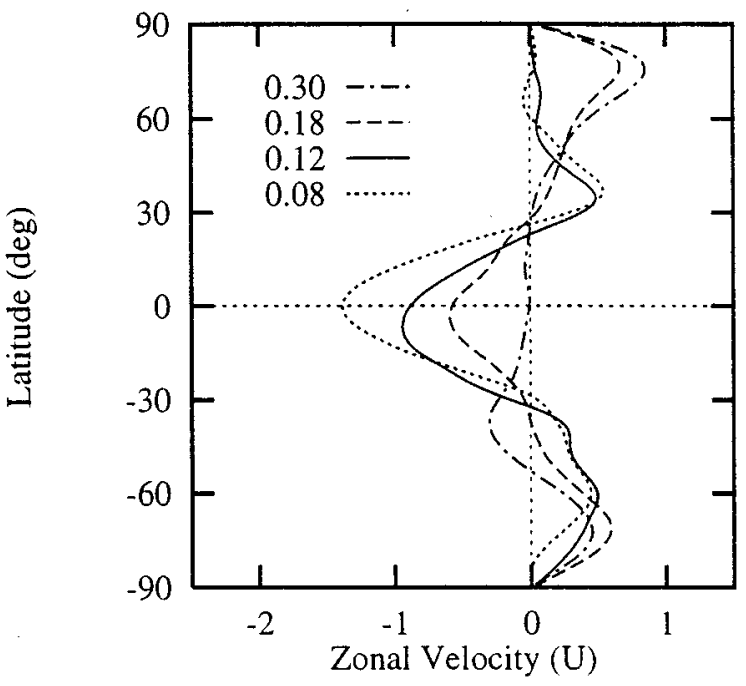

FIG. 13. The zonal velocity profiles as in Fig. 12 for several values of $H$ with fixed $\Omega$ : the $L_{D} / a$ values are shown in the legend. All other parameters are identical: $\left(p, \nu_{2 p}, n_{0}, \gamma\right)=\left(3,4.0 \times 10^{-10}, 7,18\right)$. As $H$ decreases, or as $L_{D}$ decreases, the equatorial jet intensifies.

the zonal winds decay with increasing $H$. In all our many SWE simulations, the preferred direction of the intensification is westward, and we believe this to be a general, distinguishing feature of freely evolving shallow-water systems. The fact that a number of equatorial jets in planetary atmospheres are in fact eastward, points to the conclusion that physics beyond the freely evolving shallow-water model is necessary to explain the direction of the equatorial jet.

Finally, the presence of a free surface in the runs discussed in this section allows for a clear symmetry breaking in the populations of cyclones and anticyclones. In the previous section we have shown that $\beta$ alone is not sufficient to produce an asymmetric evolution. In fact, it is not even necessary since the asymmetry has been observed on the $f$ plane. $^{19,46}$

Figure 14 clearly illustrates the cyclone/anticyclone asymmetry. Shown is the relative vorticity field in the northern hemisphere in polar-stereographic projection for a run with $\left(p, \nu_{2 p}, n_{0}, \gamma\right)=\left(3,5.0 \times 10^{-9}, 14,18\right)$, for which $L_{D} / a=0.02$ and $L_{\beta} / a=0.3$. The cyclonic vorticity is in red and the anticyclonic vorticity is in blue. Note the predominance (especially at low latitudes) and the coherence (near the pole) of blue structures as the evolution proceeds; finer temporal resolution of the early stage of evolution shows that anticyclones are able to merge more efficiently, leading to the dominance at late times in a fashion similar to the planar behavior. ${ }^{19}$ The anticyclones, which are associated with local elevations of the fluid, correspond to greater effective local value of $L_{D}$, thereby interacting and merging more efficiently with other like-signed vortices; in this way, anticyclones are able to more prominently assert themselves than their cyclonic counterparts.

In Fig. 15 we present the measures of asymmetry corresponding to the run in Fig. 14. Both the skewness and the pdf of vorticity field show a marked asymmetry toward anticyclonic vorticity. Figure 15 should be contrasted with the cor- 

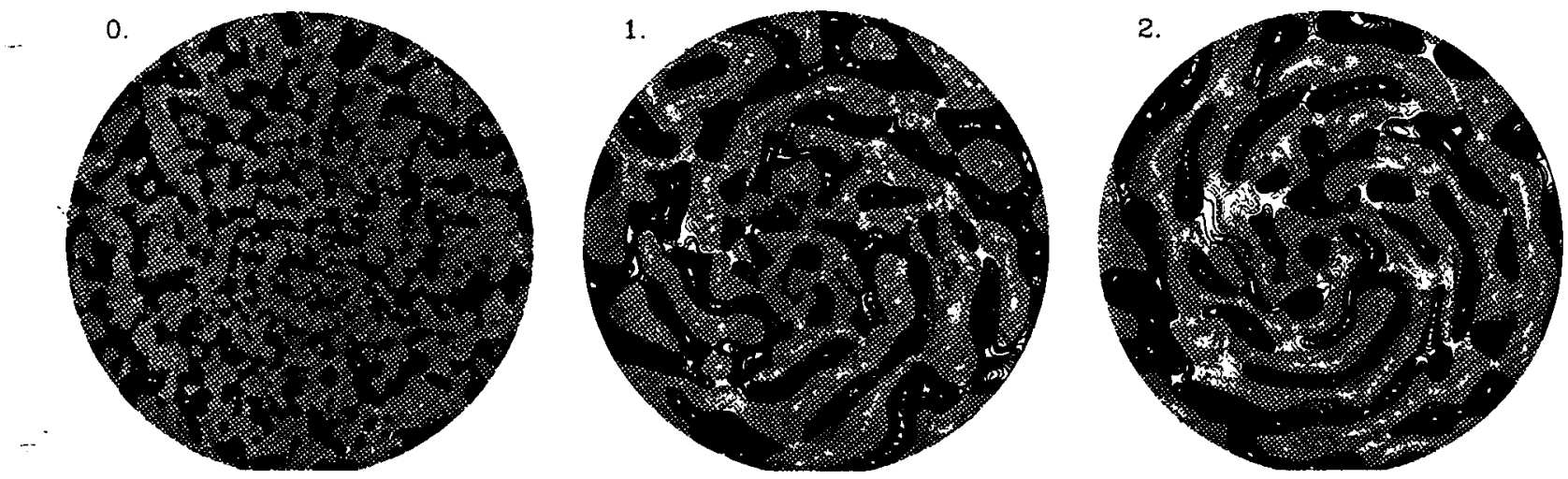

6.

7.
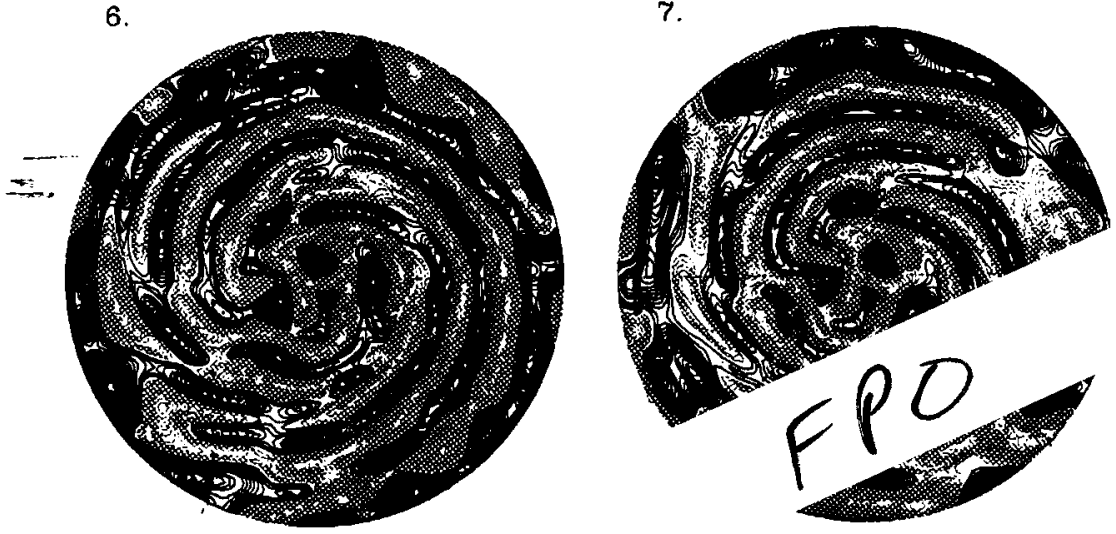

8.
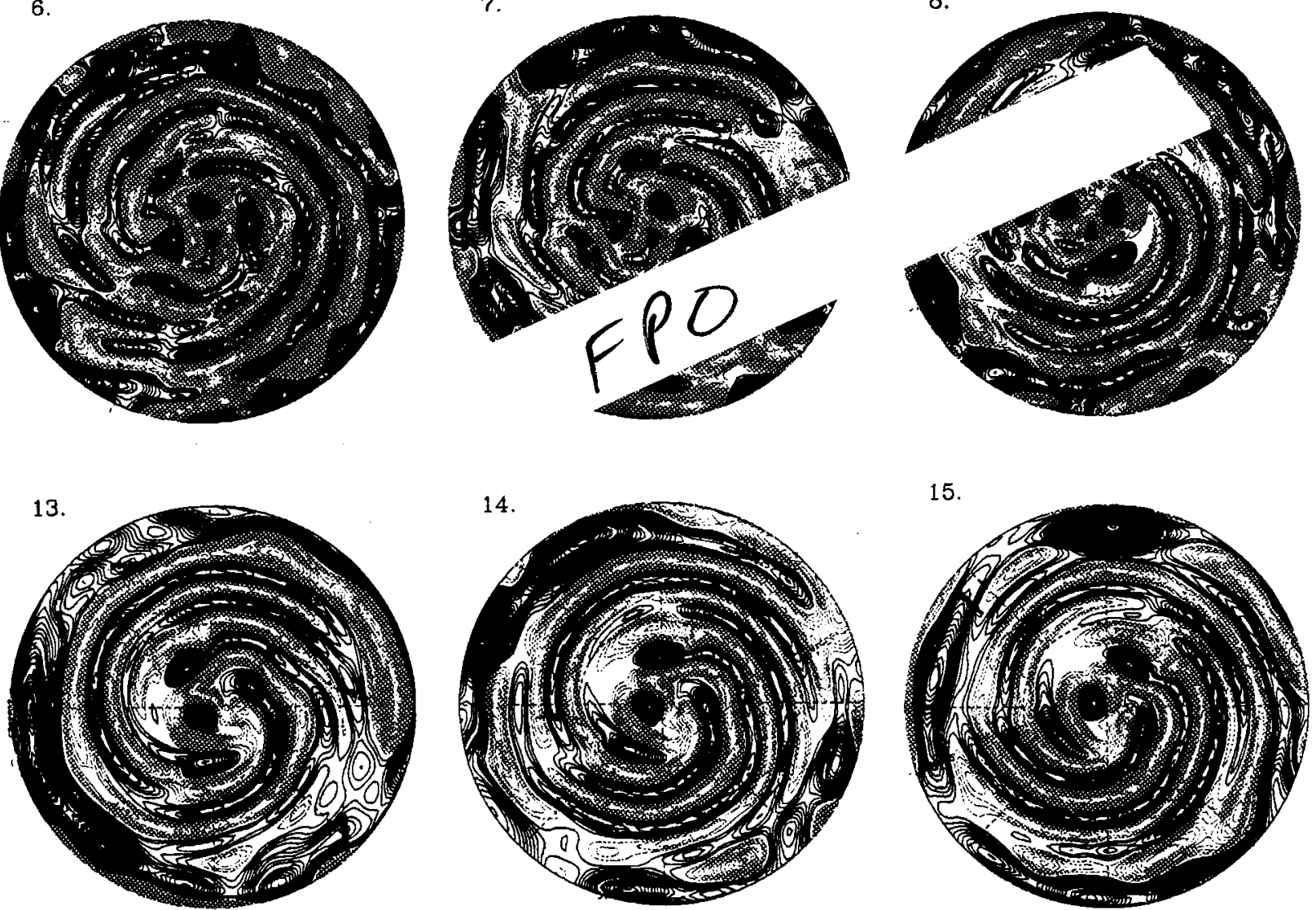

FIG. 14. The relative vorticity field in polar-stereographic projection at the North Pole. Notice the appearance of cyclone/anticyclone asymmetry: cyclonic vorticity (red) and anticyclonic vorticity (blue) for a run with $L_{\beta} / a=0.3$ and $L_{D} / a=0.02$.

responding measure given in Fig. 10 for the case where the deformation radius approaches infinity.

From the large number of simulations, we have identified the region in $(R, F)$ space, where the evolution proceeds asymmetrically. This is shown in Fig. 16, which summarizes all the shallow-water parameter values we have explored. The marks indicate initial parameter values; the "asymmetric run" is marked with an " $x$," and the "symmetric run" with a box. The asymmetry was determined by computing the vorticity skewness and the pdfs of the vorticity field. As can be seen, our runs suggest two features: (1) the relevant "asymmetry parameter" is the combination, $R B^{-1}=F^{2} R^{-1}$ (the $B=1$ line, shown as a dotted line, is also drawn for further justification of this point and is clearly not the appropriate parameter of demarcation); and (2) an initial value of $R B^{-1} \geqslant 0.13 \pm 0.04$ leads to cyclone-anticyclone symmetry breaking; the $R B^{-1}=0.13$ line in $(R, F)$ space is shown dashed in the figure. Note that physically the parameter $R B^{-1}$ corresponds to the deviation from the average height and is a measure of the ageostrophy. It is interesting to note that an $h^{\prime}=\mathscr{Q}\left(R B^{-1}\right)$ value of 0.13 is sufficient to see a 

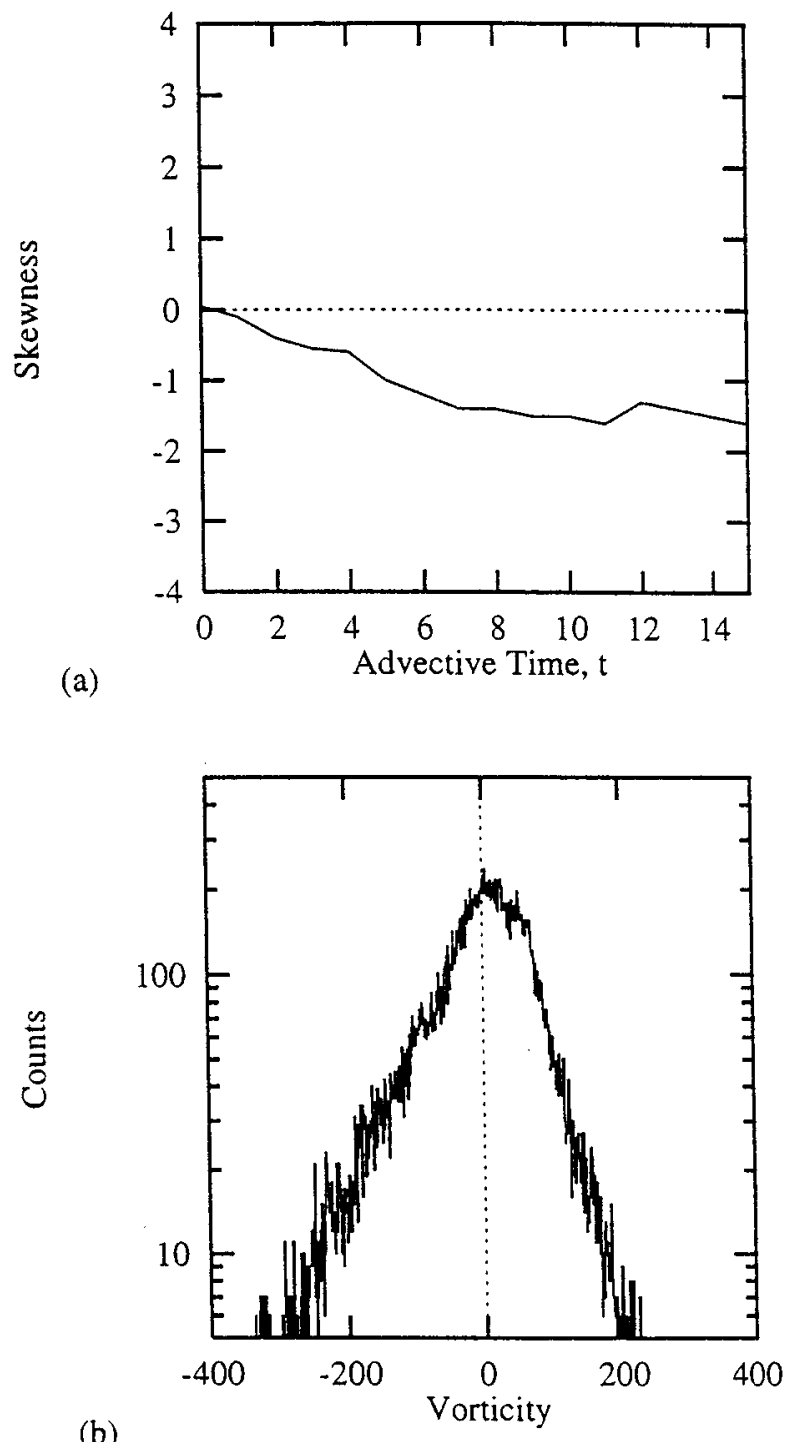

FIG. 15. The vorticity skewness time series for the run in Fig. 14(a) and the pdf of the vorticity field for the same run at $t=10$ (b). Compare with Fig. 10 for the case in which $L_{D} \rightarrow \infty$; skewness is negative, showing the dominance of anticyclonicity.

clear asymmetry, since this value does not correspond to a large surface height variation.

In summary, we have presented a careful study of parameter effects on the turbulent evolution by systematically adding complexity, beginning from the simplest possible situation. The fact that the inclusion of a finite $L_{D}$ produces zonally banded homogenized regions of potential vorticity and favors anticyclonic vortices suggest that the dynamics at play in this ultimately very simple model (no vertical structure, no thermodynamics, no forcing) may be of relevance to the atmospheres of the giant planets. We therefore conclude this survey, with a specific application to the atmosphere of Jupiter.

\section{A SIMULATION WITH JOVIAN PARAMETERS}

A number of authors have suggested that the zonal jets of the Jovian atmosphere can be understood, at least qualita-

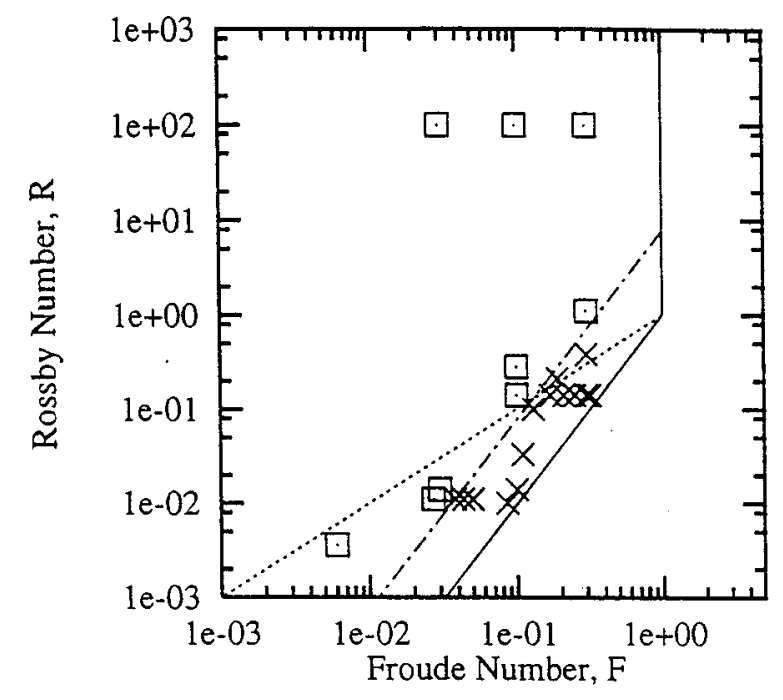

FIG. 16. The $(R, F)$ space: asymmetric runs (x's) and symmetric runs (boxes). Solid lines demarcate valid region (to their left) for shallow-water system; $B=1$ line (dotted) and $R B^{-1}=0.13$ line (dashed) are also shown.

tively, as the consequence of the nonlinear dynamics of a thin spherical shell of rotating fluid. The reader may consult the recent reviews of Rhines ${ }^{37}$ and $\mathrm{Yano}^{47}$ for more details.

However, to the best of our knowledge, all previous work along these lines has been done with forced systems and is hence liable to the criticism that the bands may be a direct product of a somewhat artificial forcing. Without denying the potentially important role of forcing, here we take a different angle of attack, along the lines of Ockham's famous razor. That is, we are interested in answering the question: how much of the observed behavior can we capture with a simpler unforced model? We believe an answer to this question will clearly discriminate between those features that are a robust spontaneous characteristic of the free dynamics and those that may be directly dependent on the currently unknown forcing.

As a specific application, results from a T170 resolution simulation with Jupiter parameters $\left(a=7.15 \times 10^{7} \mathrm{~m}\right.$, $\Omega=1.76 \times 10^{-4} \mathrm{~s}^{-1}, g=22.9 \mathrm{~m} / \mathrm{s}^{2}$, and $\left.H=2.0 \times 10^{4} \mathrm{~m}\right)$ are presented in Figs. 17 and 18. In the figures, a dimensional time unit of the Jupiter day is used. The dissipation and initial conditions for this run are $\left(p, \nu_{2 p}, n_{0}, \gamma\right)=\left(3,1.0 \times 10^{-12}, 14,18\right)$ and do not qualitatively affect the results, provided that the initial scale is small enough to allow a reasonable inverse cascade. The value of $\sqrt{g H}$ is based on observations from Voyager encounters ${ }^{48}$ and follows the shallow-water simulations of Dowling and Ingersoll ${ }^{49}$ in a channel geometry, leading to $L_{D} \sim 2 \times 10^{6} \mathrm{~m}$.

Figure 17 shows $q$ contours of this run for a long-time integration in orthographic (a) and polar-stereographic (b) projections. Many realistic features are seen: (1) there are clear banded regions of homogenized $q$, bounded in latitude by regions of high $q$ gradients (notice the multiple bunching of $q$ contours); (2) there is a large amount of eddy activity accompanying the jets-the animations clearly show many stable anticyclones that are "rolling with the winds," even at 

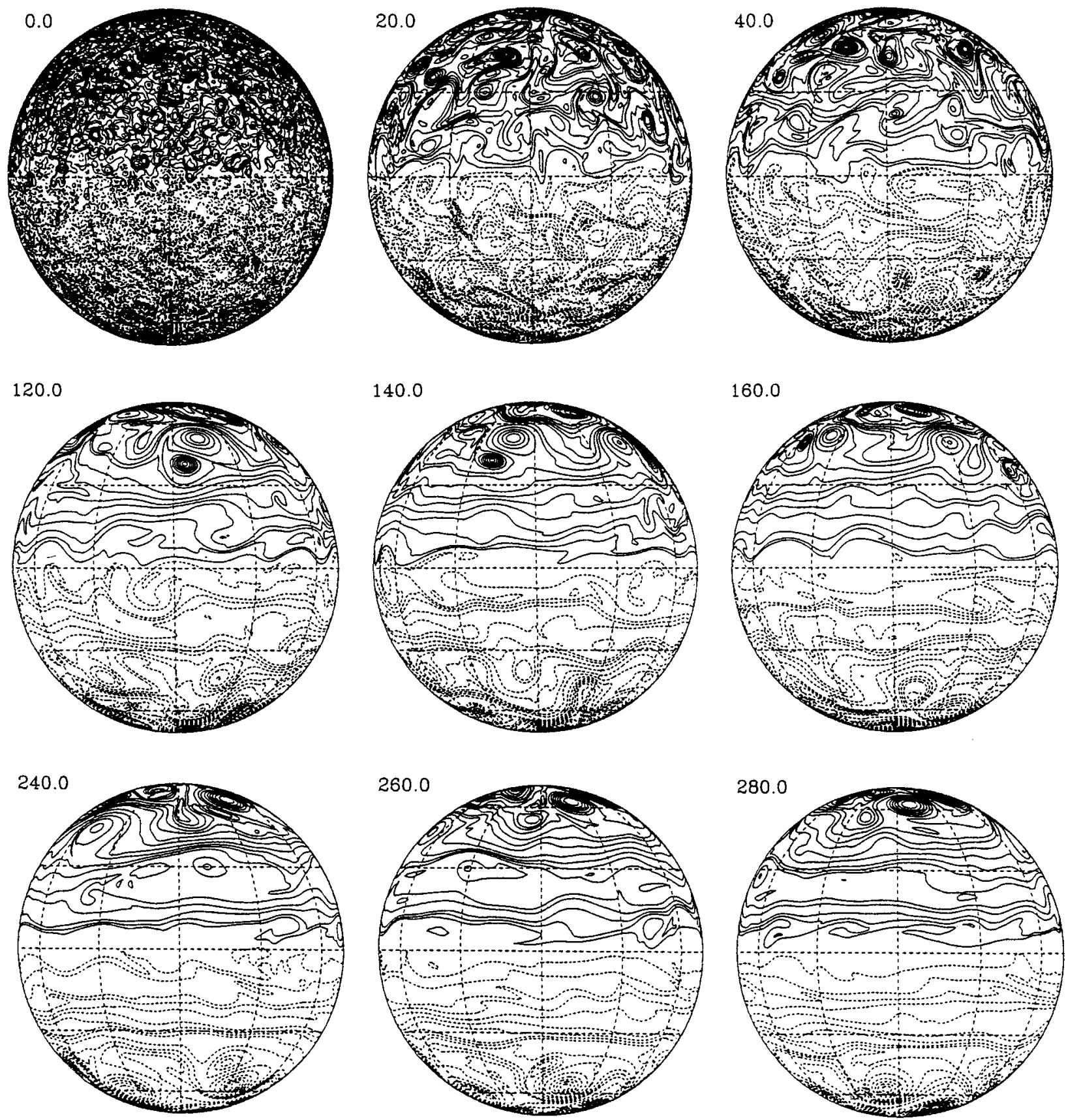

FIG. 17. A simulation with standard Jupiter parameters: $a=7.2 \times 10^{7} \mathrm{~m}, \Omega=1.8 \times 10^{-4} \mathrm{~s}^{-1}$, and $L_{D}=2 \times 10^{6} \mathrm{~m}$. Here, positive (negative) potential vorticity contours are in full (dashed) lines in (a) orthographic and (b) polar-stereographic views. Here, $t=1$ corresponds to 1 Jupiter day.

low latitudes; (3) while most of the cyclones are sheared out, anticyclones clearly persist and are much more prevailing (as indicated by the skewness of $-1.4 \pm 0.1$ in Fig. 18); (4) there is a wavy structure with high azimuthal wave number straddled by vortices at high latitude, including vortex pairs (cf. $t=120$ and $t=140$ in Fig. 17); and (5) the number and magnitude of the jets, including a strong equatorial component, are in rough agreement with the observations.

Figure 18(a) shows the zonal jets corresponding to the run in Fig. 17; the zonal wind profile can be contrasted with those presented in the previous section with smaller radius and rotation rate (cf. Figs. 12 and 13). Figure 18(b), showing the vorticity skewness, verifies the preponderance of anticyclones over cyclones.

The main discrepancy between the observed Jovian flow and the results of this simulation is that the equatorial jet is westward in our simulation, whereas on Jupiter it is eastward. Since the westward direction of the equatorial jet is a very robust feature in our shallow-water simulation (i.e., we have been unable to find parameter regimes where the equatorial jet is robustly eastward), we are forced to conclude that the eastward direction of the Jovian equatorial jet must be due to yet unknown physics that is beyond our simple, freely evolving, one-layer shallow-water model, at least at the cur- 


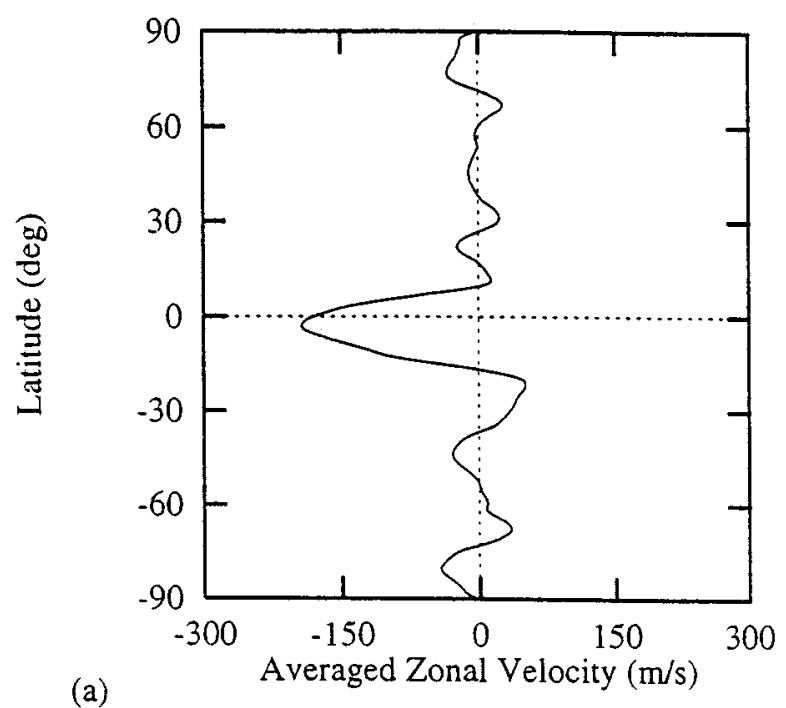

(a)

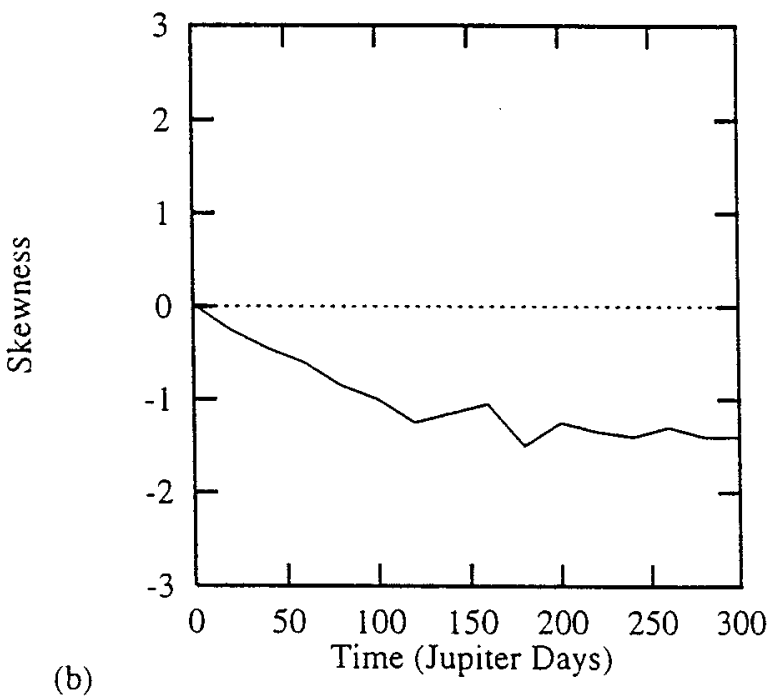

(b)

FIG. 18. Key features of Jupiter's atmosphere captured by our simple model: (a) a large number of jets in the zonal wind profile and (b) a preponderance of stable anticyclones. The equatorial jet is westward, however, in marked contrast with observations.

rent resolution. However, it is interesting to note that the equatorial jets on Uranus and Neptune are westward. ${ }^{50} \mathrm{~A}$ careful comparison of jet formation for all four Jovian giant planets will be reported elsewhere.

\section{CONCLUSION}

Three main features characterize the present work: (1) we have performed our work on a full spherical domain, with a free surface, since the spherical geometry and the presence of finite deformation radius are important to geophysical (and possibly astrophysical) applications; (2) we have presented high-resolution, long-duration, unforced simulations without initially imposing symmetry, allowing the system to evolve freely according to its own dynamics through all stages of its development; and (3) we have systematically varied only one parameter in a series of runs (totaling over 200) for a careful characterization of each parameter's influence on the evolution.

As a result, a number of phenomena previously not well emphasized or observed have been demonstrated. First, the spectral evolution was shown to be highly dependent on the initial conditions, with very sensitive behavior attributed to the initially most-energetic scale, as well as to the choice of dissipation. Also, in the absence of rotation and in the limit of infinite deformation radius $L_{D}$, the end state was found to be in a vortical quadrupole configuration.

Second, in the presence of rotation, vortices undergo continuous inelastic interactions (i.e., various degrees of mergers and straining $)^{51}$ in the poleward direction-evolving finally toward a solid-body rotation end-state. At low rotation rates, some zonal jets form, but are not steady, since vortical structures are not well confined in the meridional direction. At high rotation rates (and $L_{D} \rightarrow \infty$ ), the evolution is dominated by a somewhat surprising circumpolar vortices. This last result independently confirms the earlier calculation of Yoden and Yamada ${ }^{9}$ over a much broader range of simulation conditions.

Third, the addition of a free surface (and thus of a finite $L_{D}$ ) leads to a number of new and interesting behaviors. A finite $L_{D}$ provides a second inverse cascade arrest mechanism, and is responsible for the absence of polar vortices in spherical shallow-water turbulence, even at high rotation rates. Furthermore, a finite value of $L_{D}$ leads to the formation of robust, zonally elongated structures with a jet profile containing an intensifying, westward, equatorial component-increasing as $L_{D}$ is decreased.

In addition, we find that, as in the planar case, anticyclonic vortices are favored over cyclonic ones and that the asymmetry appears to be controlled by the parameter $R B^{-1}$. Although $\beta$ is not required for asymmetry, it does seem to enhance the asymmetry, in that anticyclones are always the preferred vortical structures in the equatorial regions, where the value of $\beta$ is large.

Finally, we have performed a detailed simulation with Jovian parameters. While it is clear that many of the key qualitative features of Jupiter's atmosphere can be captured by a free-evolving shallow-water system, at least one serious qualitative discrepancy has clearly emerged, namely the direction of equatorial winds. We hope future studies will shed new light on this matter.

\section{ACKNOWLEDGMENTS}

This research was supported in part by the National Science Foundation (NSF) National Young Investigator Award to LMP. The computations were performed on the CRAYYMP/C90 at the Pittsburgh Supercomputing Center, which is supported by the NSF. The original version of the global shallow-water code was provided to us by J. Hack and R. Jakob at NCAR. The authors also wish to acknowledge helpful discussions with R. Pelz during the early stages of this work and thank the anonymous referees for helpful suggestions. 
${ }^{1}$ J. C. McWilliams, "The emergence of isolated coherent vortices in turbulent flow," J. Fluid Mech. 146, 21 (1984).

${ }^{2}$ S. Kida, "Numerical simulation of two-dimensional turbulence with highsymmetry," J. Phys. Soc. Jpn. 54, 2840 (1985).

${ }^{3}$ M. E. Brachet, M. Meneguzzi, and P. L. Sulem, "Small scale dynamics of high Reynolds number two-dimensional turbulence," Phys. Rev. Lett. 57, 683 (1986).

${ }^{4}$ R. Benzi, S. Patarnello, and P. Santangelo, "Self-similar coherent structures in two-dimensional decaying turbulence,'” J. Phys. A Math. Gen. 21, 1221 (1988)

${ }^{5}$ P. Santangelo, R. Benzi, and B. Legras, "The generation of vortices in high-resolution, two-dimensional decaying turbulence and the influence of initial conditions on the breaking of self-similarity," Phys. Fluids A 1, 1027 (1989).

${ }^{6}$ J. C. McWilliams, "The vortices of two-dimensional turbulence," J. Fluid Mech. 219, 361 (1990).

${ }^{7}$ R. Benzi, M. Briscolini, M. Colella, and P. Santangelo, "A simple point vortex model for two-dimensional decaying turbulence," Phys. Fluids A 4, 1036 (1992)

${ }^{8} \mathrm{C}-\mathrm{M}$. Tang and S. Orszag, "Two-dimensional turbulence on the surface of a sphere," J. Fluid Mech. 87, 305 (1978).

${ }^{9}$ S. Yoden and M. Yamada, "A numerical experiment on two-dimensional decaying turbulence on a rotating sphere," J. Atmos. Sci. 50, 631 (1993).

${ }^{10}$ D. G. Dritschel, "The vortex property of two-dimensional turbulence," Phys. Fluids A 5, 984 (1993).

${ }^{11}$ P. B. Rhines, "Waves and turbulence on the $\beta$-plane,'” J. Fluid Mech. 69, 417 (1975)

${ }^{12}$ P. B. Rhines, “Geostrophic turbulence,” Annu. Rev. Fluid Mech. 11, 404 (1979).

${ }^{13}$ M. E. Maltrud and G. K. Vallis, "Energy spectra and coherent structures in forced two-dimensional and beta-plane turbulence," J. Fluid Mech. 228, 321 (1991).

${ }^{14} \mathrm{G}$. K. Vallis and M. E. Maltrud, "Generation of mean flows and jets on a beta plane and over topography," J. Phys. Oceanogr. 23, 1346 (1993).

${ }^{15}$ G. P. Williams, "Planetary circulations: 1 barotropic representations of Jovian and terrestrial turbulence," J. Atmos. Sci. 35, 1399 (1978).

${ }^{16}$ C. Basdevant, B. Legras, R. Sadourny, and M. Beland, "A study of barotropic model flows: Intermittency, waves and predictability," J. Atmos. Sci. 38, 2305 (1981).

${ }^{17}$ M. Farge and R. Sadourny, "Wave-vortex dynamics in rotating shallow water," J. Fluid Mech. 206, 433 (1990).

${ }^{18}$ M. A. Spall and J. C. McWilliams, "Rotational and gravitational influences on the degree of balance in the shallow water equations," Geophys. Astrophys. Fluid Dyn. 64, 1 (1992).

${ }^{19}$ L. M. Polvani, J. C. McWilliams, M. A. Spall, and R. Ford, “The coherent structures of shallow-water turbulence: Deformation-radius effects, cyclone/anticyclone asymmetry and gravity-wave generation," Chaos $\mathbf{4}$, 177 (1994)

${ }^{20}$ Indeed, to the best of our knowledge, a detailed and comprehensive sensitivity study in fully turbulent, decaying situations has never been performed, particularly for the spherical geometry.

${ }^{21}$ J. Pedlosky, Geophysical Fluid Dynamics (Springer-Verlag, Berlin, 1987).

${ }^{22}$ B. Cushman-Roisin and B. Tang, "Geostrophic turbulence and emergence of eddies beyond the radius of deformation," J. Phys. Oceanogr. 20, 97 (1990).

${ }^{23}$ The $\langle\cdot\rangle$ operator denotes a spatial average $1 / 4 \pi \int_{0}^{2 \pi} \int_{-\pi / 2}^{\pi / 2}(\cdot) \cos \vartheta d \vartheta d \lambda$.

${ }^{24}$ J. J. Hack and R. Jakob, NCAR Technical Note NCAR/TN-343+STR, 1992.

${ }^{25}$ Computations were performed on a 16-processor CRAY-YMP/C90 with a parallelized code.
${ }^{26}$ In the spherical geometry, the total wave number $n$ plays the analogous role of $k$ in the planar geometry.

${ }^{27}$ G. J. Haltiner and R. T. Williams, Numerical Prediction and Dynamic Meteorology (Wiley, New York, 1980)

${ }^{28}$ W. H. Matthaeus, W. T. Stribling, D. Martinez, S. Oughton, and D. Montgomery, "Selective decay and coherent vortices in two-dimensional incompressible turbulence," Phys. Rev. Lett. 66, 2731 (1991).

${ }^{29} \mathrm{~A}$ fiducial cut in spectral range is made for the slope determination using a "best-fit" procedure on the compensated spectra; an average of the slopes over three frames and three runs are taken. The precise values of the slopes are sensitive to the range examined; however, the emphasis here is on the trends.

${ }^{30}$ A. Mariotti, B. Legras, and D. G. Dritschel, "Vortex stripping and the erosion of coherent structures in two-dimensional flows," Phys. Fluids 6, 3954 (1994)

${ }^{31}$ H. B. Yao, N. J. Zabusky, and D. G. Dritschel, “'High gradient phenomena in two-dimensional vortex interactions," Phys. Fluids 7, 539 (1995).

${ }^{32}$ In this case, the vortex size distribution is different at $t=0$ due to the difference in $\gamma$.

${ }^{33} \mathrm{~V}$. Borue, "Spectral exponents of enstrophy cascade in stationary twodimensional homogeneous turbulence,"' Phys. Rev. Lett. 71, 3967 (1993).

${ }^{34}$ G. F. Carnevale, J. C. McWilliams, Y. Pomeau, J. B. Weiss, and W. R. Young, "Evolution of vortex statistics in two-dimensional turbulence," Phys. Rev. Lett. 66, 2735 (1991).

${ }^{35}$ P. S. Marcus, "Vortex dynamics in a shearing zonal flow," J. Fluid Mech. 215, 393 (1990)

${ }^{36}$ The spectrum used in Ref. 9 is qualitatively similar to the one used here; their spectral peak location corresponds to a slightly larger $n_{0}$.

${ }^{37}$ P. B. Rhines, "Jets," Chaos 4, 313 (1994).

${ }^{38}$ This behavior is also observed in the full shallow-water cases.

${ }^{39}$ Recall that a vortex is cyclonic when its vorticity has the same sign as the planetary rotation, and anticyclonic otherwise.

${ }^{40}$ M. V. Nezlin and E. N. Snezhkin, Rossby Vortices, Spiral Structures and Solitons (Springer-Verlag, Berlin, 1993).

${ }^{41}$ L. M. Polvani, N. J. Zabusky, and G. R. Flierl, "Two-layer geostrophic vortex dynamics. Part 1. Upper layer $V$-states and merger,'” J. Fluid Mech. 205, 215 (1989)

${ }^{42}$ V. D. Larichev and J. C. McWilliams, "Weakly decaying turbulence in an equivalent barotropic model," Phys. Fluids A 3, 938 (1991).

${ }^{43}$ D. W. Waugh, Ph.D. thesis, Cambridge University, 1992.

${ }^{44}$ In the nondivergent cases initial balancing is not required, since gravity waves are not present.

${ }^{45} \mathrm{G}$. Browning, A. Kasahara, and H-O. Kreiss, "Initialization of primitive equations by the bounded derivative method," J. Atmos. Sci. 37, 1424 (1980).

${ }^{46}$ M. Arai and T. Yamagata, "Asymmetric evolution of eddies," Chaos 4, 163 (1994).

${ }^{47}$ J.-I. Yano, "A critical review on the dynamics of Jovian atmospheres," Chaos 4, 287 (1994).

${ }^{48}$ A. P. Ingersoll, "Atmospheric dynamics of the outer planets," Science 248, 308 (1990).

${ }^{49}$ T. E. Dowling and A. P. Ingersoll, "Jupiter's Great Red spot as a shallow water system," J. Atmos. Sci. 46, 3256 (1989).

${ }^{50} \mathrm{R}$. Beebe, "Characteristic zonal winds and long-lived vortices in the atmosphere of the outer planets," Chaos 4, 113 (1994).

${ }^{51}$ D. G. Dritschel and D. W. Waugh, "Quantification of the inelastic interaction of two asymmetric vortices in two-dimensional vortex dynamics," Phys. Fluids A 4, 1737 (1992). 